\title{
Distinctions among electroconvulsion- and proconvulsant-induced seizure discharges and native motor patterns during flight and grooming: Quantitative spike pattern analysis in Drosophila flight muscles
}

\author{
Jisue $\mathrm{Lee}^{1 \dagger}$, Atulya lyengar ${ }^{1,2 \dagger}$, Chun-Fang $\mathrm{Wu}^{1,2}$ *
}

\begin{abstract}
In Drosophila, high-frequency electrical stimulation across the brain triggers a highly stereotypic repertoire of spasms known as electroconvulsive seizures (ECS). The distinctive ECS spiking discharges manifest across the nervous system and can be stably assessed throughout the seizure repertoire in the large indirect flight muscles (DLMs). ECS discharges in DLMs have been extensively used to monitor seizure activities, notably in stress ('bang')-sensitive mutants. However, the relationships between ECS-spike patterns and native motor programs, including flight and grooming, are not known and their similarities and distinctions remain to be characterized. We employed quantitative spike pattern analyses including: 1) overall firing frequency, 2) spike timing between contralateral fibers, and 3) short-term variability in spike interval regularity $\left(\mathrm{CV}_{2}\right)$ and instantaneous firing frequency $\left(\mathrm{ISl}^{-1}\right)$ to reveal distinctions amongst ECS discharges, flight and grooming motor patterns. We then examined DLM firing phenotypes in well-established mutants in excitatory cholinergic (Cha), inhibitory GABAergic $(R d l)$ and electrical $(S h a k B)$ synaptic transmission. The results provide an initial glimpse on the vulnerability of individual motor patterns to perturbations of respective synaptic transmission systems. We found marked alterations of ECS discharge spike patterns in terms of either seizure threshold, spike frequency or spiking regularity. In contrast, no gross alterations during grooming and only a minor reduction of firing frequency during Rdl mutant flight were observed, suggesting a role for GABAergic modulation of flight motor programs. Picrotoxin (PTX), a known pro-convulsant that inhibits $\mathrm{GABA}_{\mathrm{A}}$ receptors, induced DLM seizure patterns that displayed some features, e.g. left-right coordination and $\mathrm{ISl}^{-1}$ range, that could be found in flight or grooming, but distinct from ECS discharges. Our results indicate that these quantitative techniques may be employed to reveal overlooked relationships among aberrant motor patterns and native DLM motor programs in genetic and pharmacological analyses of underlying cellular and neural circuit function.
\end{abstract}

\section{Keywords}

Dorsal Longitudinal Muscle - Poincarè Plot — Instantaneous Firing Frequency — Instantaneous Coefficient of Variation

${ }^{1}$ Department of Biology ${ }^{2}$ Interdisciplinary Graduate Program in Neuroscience

University of lowa, lowa City, IA, 52242, United States

*Corresponding author: chun-fang-wu@uiowa.edu. ${ }^{\dagger}$ Equal Contribution

\section{Introduction}

For nearly half a century, Drosophila mutants have opened the avenue to the mechanistic elucidation of how genetic variations contribute to the physiological basis of epileptiform behaviors and related seizure disorders [1,2,3]. Early mutants were isolated on the basis of seizures triggered by stressors such as high temperature (e.g. shibire [4], seizure [5]) or mechanical shock (e.g. bang-senseless, easily shocked, knockdown, and bang-sensitive [6]), while other mutants displayed spontaneous spasms (e.g. Shudderer [7]). Mutational studies have led to identifications of individual genes associated with seizure disorders, including those encoding for or regulating ion channels $[8,9,10,11]$, ionic transport and distribution $[12,13,14]$, and enzymes related to energy metabolism $[15,16,17,18]$. Different adult preparations [19, 20, 21, 22] have been employed to monitor seizure activity in the nervous system. In particular, the indirect flight muscles, dorsal longitudinal muscles (DLMs), have been most widely utilized as a readout of aberrant motor activities, especially during seizure discharges. High-frequency stimulation across the brain can trigger an electroconvulsive seizure (ECS [20]) with a highly stereotypic repertoire consisting of an initial spike 
discharge (ID), a period of paralysis, and a second delayed spike discharge (DD [21]). This seizure repertoire is modified in specific manners characteristic of individual seizure mutants [21,22]. This line of studies demonstrates that the large DLM can provide convenient but reliable readouts for epileptiform and other patterned neural activities generated in the central nervous system (see [20, 23, 21, 22, 24, 25]).

A number of activity patterns driven by the innervating motor neuron (DLMn) have been characterized in detail, including flight, grooming, courtship and giant-fiber mediated jump-and-flight escape. During flight, the DLM fibers (six on each side) undergo myogenic, stretch-activated, isometric contractions to generate tension in phase with the oscillation of thorax case to power wing depression during the wing beat cycles $(\sim 200 \mathrm{~Hz},[26,27])$. DLM action potentials, evoked by DLM motor neuron (DLMn) spikes, fire continuously and rhythmically at approximately $5 \mathrm{~Hz}$ [28, 29] solely to facilitate $\mathrm{Ca}^{2+}$ influx required for continued oscillation in muscle tension $[30,31]$. In contrast, discrete individual spikes are associated with giant-fiber pathway activation [32, 33, 34, 35, 36], while distinct spike bursting activities are associated with grooming [34] and courtship song production [37].

In this report, we quantitatively describe the firing patterns of the DLM during electroconvulsion-induced seizure discharges versus the native spiking patterns during flight and grooming. In addition to ensemble statistics, we analyzed bilateral coordination of motor activities, as well as the local temporal features of spike trains, including instantaneous firing rate, short-term firing regularity, and timing relationships among adjacent spikes.

We further examined how activity patterns characteristic of each motor programs were affected upon genetic or pharmacological manipulations. We studied well-characterized mutants of major neurotransmission systems known to have clear alterations in physiology and behavioral expression: 1) picrotoxin (PTX), a non-competitive antagonist of the $\mathrm{GABA}_{\mathrm{A}}$ receptor [38]; 2) a related receptor mutant, Resistant to dield$\operatorname{rin}(R d l)$ that encodes the $\mathrm{GABA}_{\mathrm{A}}$ receptor $\alpha$ subunit [39]; 3) Choline acetyltransferase (Cha) mutants with reduced acetylcholine (ACh) synthesis [40, 41]; and 4) mutants of ShakB, encoding an innexin gap junction protein required for transmission at electrical synapses [42]. These treatments revealed clear quantitative distinctions between the seizure discharge patterns evoked by ECS and that induced by GABA receptor blockade via PTX. Thus, the quantitative approaches undertaken here may be applicable to the study of additional aberrant DLM spike discharges to explore their potential association with or distinction from the endogenous spike patterns during normal motor activities.

\section{Methods}

\section{Fly Strains}

Drosophila melanogaster strains were kept in standard glass vials containing cornmeal medium [43] and reared at room temperature $\left(23^{\circ} \mathrm{C}\right)$. Adult flies used for the experiments were
3-12 days old. Wild-type flies were of the strain Canton $S$ (CS). Synaptic transmission mutants examined include those alleles defective in Cha encoding a Choline Acetyltransferase (Cha ${ }^{t s 2}$ [44]), Rdl encoding the $\mathrm{GABA}_{\mathrm{A}}$ receptor a subunit $\left(R d l^{M D-R R}\right.$, [39]), and $S h a k B$ encoding the structural subunit of an innexin (ShakB ${ }^{2}$ [45]; ShakB Pas [42], also known as $\left.S h a k B^{3},[46]\right)$. Data from male and female individuals were pooled, as previous results have indicated that DLM firing properties are not generally different between sexes during flight or seizure activity [21, 22, 27].

\section{Tethered Preparation and DLM Recording}

The fly tethering procedure used for DLM recordings have been described in detail previously [21, 22, 27]. Flies were briefly cold- or ether-anesthetized and glued to a tungsten pin using cyanoacrylate or nitrocellulose based glue. Flies were allowed to recover from anesthesia for at least $30 \mathrm{~min}$ prior to recording. All recordings were done at room temperature $(\sim$ $23^{\circ} \mathrm{C}$ ).

Electrolytically sharpened tungsten electrodes were used to access DLM spikes, with an electrode inserted into the dorsal-most fiber (DLMa, 45a, c.f. [47]), and a similarly constructed electrode inserted into the abdomen for reference. Signals were amplified by an AC amplifier (either DAM5A, World Precision Instruments, New Haven CT; or AM Systems Model 1800, Carlsborg, WA) and digitized via a data acquisition card at a sampling rate $>10 \mathrm{kHz}$ (Digidata 1200, Axon Instruments, or USB 6210, National Instruments). Spike detection and analysis were done in PClamp 6, LabVIEW 8.6 and in MATLAB r2017b using custom-written scripts.

\section{Electroconvulsive Stimulation}

Electroconvulsive stimulation was delivered across the brain via tungsten electrodes inserted into each eye, and consisted of a train of $0.1-\mathrm{ms}$ pulses delivered at $200 \mathrm{~Hz}$ at a specified voltage $(30-100 \mathrm{~V})$ and train duration $(0.5-4 \mathrm{~s})$. Seizure susceptibility of individual flies was examined for each genotype (Figure 6) using five different intensity levels 1 - 5 (stimulus voltage, train duration): 1 . (50 V, $0.5 \mathrm{~s}) ; 2$. (50 V, $1.0 \mathrm{~s}) ; 3$. $(50 \mathrm{~V}, 2.0 \mathrm{~s})$; 4. (100 V, $2.0 \mathrm{~s})$; and 5. (100 V $4.0 \mathrm{~s})$. Each level corresponds to a progressive doubling of stimulation intensity [22]. When appropriate, after the electroconvulsive stimulation, test pulses sufficient to directly activate the giantfiber neuron ( $24 \mathrm{~V}$ or higher, $0.1 \mathrm{~ms}$ duration, delivered at $1 \mathrm{~Hz}$ ) were applied to examine synaptic transmission along giant-fiber pathway.

\section{Pharmacology}

Picrotoxin (PTX) was used to block ionotropic GABAergic signaling [21]. Two methods were employed to administer PTX systemically in intact, tethered flies: 1) small droplet applied on an eye incision ('eye drop'), and 2) a fixed volume injection into the dorsal vessel ('DV inejection'). For eye drop application, a stimulation electrode was advanced to penetrate the basement membrane of the retina and then retracted. A small drop of $1 \mathrm{mM}$ PTX solution dissolved in $0.6 \% \mathrm{NaCl}$ 
solution (or Control $\mathrm{NaCl}$ solution without PTX) was applied to the incision and was allowed to diffuse to the hemolymph. The DV injection procedure was adapted from Howlett \& Tanouye [48]. A filamented glass electrode $(1.0 \mathrm{~mm}$ OD, $0.58 \mathrm{~mm}$ ID, A-M Systems \#601500) was pulled in a BrownFlaming electrode puller (P87, Sutter Instruments, Novato, CA). After loading the distal end of the electrode with PTX solution, capillary action consistently drew $0.33 \mu \mathrm{l}$ of solution to the tip. To inject the loaded solution, the electrode tip was broken and was inserted into the dorsal vessel [47] of the tethered fly. The solution was then injected using air pressure delivered manually through a syringe.

\section{Spike Train Analysis}

Firing rate was analyzed using two measures: instantaneous firing frequency and overall spiking rate over a specified time window of interest. The instantaneous firing frequency was defined as the reciprocal of the inter-spike interval (ISI) measured in seconds, between successive spikes [49, 50], referred to as $\mathrm{ISI}^{-1}$ with units of $\mathrm{Hz}$. For sequential ISI ${ }^{-1} \mathrm{~s}$ in a spike train, the occurrence of the first spike is used to mark the temporal location of the particular inter-spike interval. The overall spike rate was defined as the total spike count during the specified time window divided by its duration. Poincaré trajectories (Figure 4, Figure 7 and Figure 9) were constructed by plotting the time series of ISI $^{-1}$ within a spike train, with each ISI $^{-1}$ against the ISI $^{-1}$ of the subsequent interval, i.e. $\mathrm{ISI}^{-1}{ }_{i}$ vs. $\mathrm{ISI}^{-1}{ }_{i+1}$. Therefore for a spike train of $n$ spikes, there will be $n-1$ data points in the Poincare trajectory.

To quantify the regularity of ISIs within a spike train, the coefficient of variation, $\mathrm{CV}$, defined as the standard deviation of ISIs divided by the mean ISI, has been extensively used (e.g. [51]). However, to accurately estimate variability, the average firing frequency of the spike train is usually assumed to be stationary over a sufficiently long period of firing for which the $\mathrm{CV}$ is computed. To assess variability in spike trains with varying firing frequency over relatively short time periods, Holt and associates [52] developed $\mathrm{CV}_{2}$ which estimates variability between adjacent spike intervals. The $\mathrm{CV}_{2}$ is computed for each spike sequentially along the spike train from each pair of two adjacent intervals $\mathrm{ISI}_{i}$ and $\mathrm{ISI}_{i+1}$ as:

$$
\mathrm{CV}_{2}=2\left|\mathrm{ISI}^{-1}{ }_{i}-\mathrm{ISI}^{-1}{ }_{i+1}\right| /\left(\mathrm{ISI}^{-1}{ }_{i}+\mathrm{ISS}^{-1}{ }_{i+1}\right)
$$

For cases of spike trains with stationary average firing rates over time, estimates of variability derived from $\mathrm{CV}$ and $\mathrm{CV}_{2}$ are in close agreement [52]. For each spike train, the scatterplot of the $\mathrm{ISI}^{-1}$ versus $\mathrm{CV}_{2}$ documents the local firing frequency (or ISI-1), and firing frequency variability $\left(\mathrm{CV}_{2}\right)$ around individual spikes (represented as points in the 2-D plot). After connecting temporally adjacent spike-points, the trajectory thus traces the history of the spike-interval variability along with the sequential instantaneous spike firing frequencies. Note that the plot of the $\mathrm{ISI}^{-1}$ versus $\mathrm{CV}_{2}$, may be derived from the Poincaré trajectory, which registers sequentially the ISI $^{-1}$ before and after each individual spike throughout the same spike train.
For assessment of the general trends and for clarity in presentation, we applied a running average filter to smoothen the raw $\mathrm{ISI}^{-1}$ vs. $\mathrm{CV}_{2}$ plots. We verified that filter window sizes ranging from 3-9 pts provided qualitatively similar results without severely distorting major features of the trajectory for the three motor programs examined here. A 6-spike window was applied in $\mathrm{ISI}^{-1}$ vs. $\mathrm{CV}_{2}$ plots because it appropriately captures the characteristics for both sustained flight and short grooming bouts (Supplemental Figure 1).

The filtered $\mathrm{ISI}^{-1}$ vs. $\mathrm{CV}_{2}$ plots also enabled us to obtain smoothened 'overall' trajectories for the different motor patterns based on the ensemble of spike trains from recorded individuals, even though each train could differ somewhat in numbers of spikes. Briefly, durations of individual spike trains were normalized, such that ' 0 ' represented the initial spike and ' 1 ' the last spike, with the relative temporal locations for the rest of spikes reassigned proportionally along the normalized unitary duration. Thus, an overall average trajectory of a specific motor pattern could be constructed based on the collective temporal information from an ensemble of spike trains, i.e. to determine the averaged $\mathrm{ISI}^{-1}$ vs. $\mathrm{CV}_{2}$ values, or $\left(\mathrm{ISI}^{-1}, \mathrm{CV}_{2}\right)$, along the unitary duration. For a spike train in the ensemble, each rescaled ISI segment still carries the same $\left(\mathrm{ISI}^{-1}, \mathrm{CV}_{2}\right)_{i}$ associated with the $i$ th spike. The averaging process for the ensemble (ISI ${ }^{-1}, \mathrm{CV}_{2}$ ) was carried out with an increment of 0.01 (bin size) along the normalized duration. The resulting variation of $\left(\mathrm{ISI}^{-1}, \mathrm{CV}_{2}\right)$ along the normalized the time base, or unitary duration, was re-plotted in the 2-D graph with ISI ${ }^{-1}$ vs. $\mathrm{CV}_{2}$ coordinates. The range of number of ISIs for this treatment was between $\sim 60$ and $\sim 300$ for DD, $\sim 400$ and $\sim 1200$ for flight, and 20 and $\sim 500$ for grooming spike trains. The outlier spike trains were eliminated from the calculation (mostly occurred in grooming).

The bilateral phase $(\theta)$ relation between individual spikes from the left and right DLM units was calculated as the proportion of the time point within the contralateral inter-spike interval that the ipsilateral unit generated a spike, i.e. $\theta$ ranges from 0 to $1[28,53]$.

\section{Results}

Three Identified Motor Programs Driving DLM Activity: Electroconvulsive Seizure Discharges, Flight and Grooming

High frequency stimulation across the brain triggers a previously described ECS repertoire in wild-type (WT) and different mutants [20,21], which consists of an initial discharge (ID) of DLM spikes corresponding to an initial spasm, a period of paralysis where conduction fails along the giant fiber (GF) jump-and-flight escape pathway, followed by a delayed discharge (DD) of DLM spikes, and finally recovery of GF pathway transmission and voluntary motor behaviors. The present study focuses on DD spike patterns since quantitative aspects of DLM spiking during the ID have been characterized $[17,27]$ whereas spike patterning during the DD has received less attention [22]. We noted that flies often display a 'wings- 

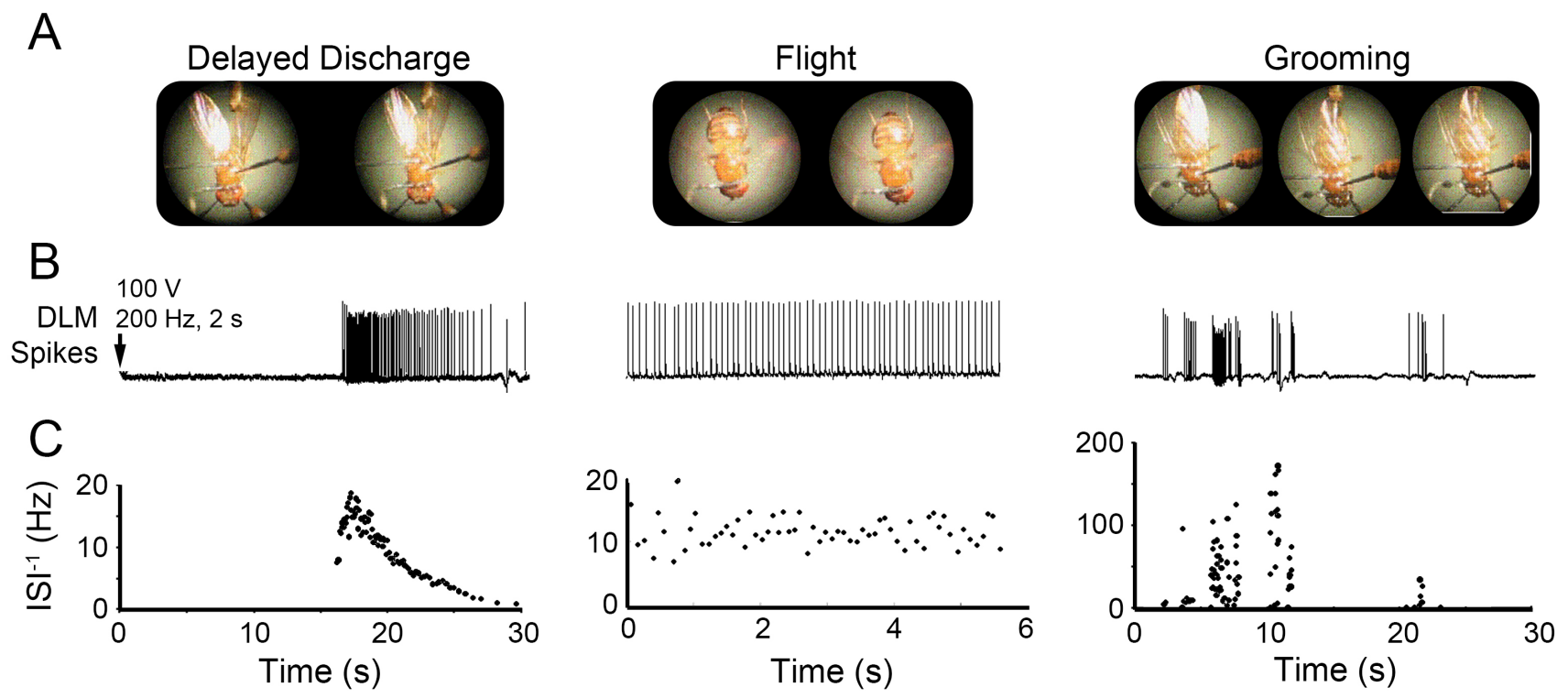

Figure 1. Wing posture and corresponding DLM spiking activity during delayed seizure discharges, flight and grooming.

(A) Representative consecutive video frames (50 ms apart) of tethered fly behavior taken during the respective motor programs. During the delayed seizure discharges (DD), wings were often held in an 'up' position, although brief wing buzzes were sometimes observed. During flight the wings beat at approximately $200 \mathrm{~Hz}$ [27]. Grooming bouts were often characterized by the fly depressing its wing to enable leg sweeps over the dorsal wing surface. (B) Example traces of DLM spiking corresponding to the behaviors in (A). Arrow indicates timing of electroconvulsive stimulation triggering delayed discharges. Note the rhythmic DLM spiking activity during flight and bursts of activity associated with grooming behavior. (C) Plots of the instantaneous firing frequency (inverse of inter-spike intervals, ISI $^{-1}$ ) versus time corresponding with the respective traces in (B). The ISI ${ }^{-1}$ s observed during grooming bouts were an order of magnitude higher than firing observed during either DD or flight.

up' posture during DD (Figure 1A, Supplemental Video 1, cf. [21]). During flight, the wings beat at approximately $200 \mathrm{~Hz}$ [54, 55, 27], and during grooming, wing depression facilitates leg sweeping across the wing (Figure 1A, [34, 56, 57]). We observed characteristic patterns of DLM spiking corresponding with these behaviors (Figure 1B), each with distinct profiles of instantaneous firing frequency (defined as the reciprocal of each inter-spike interval, ISI ${ }^{-1}$ with units $\mathrm{Hz}$, Figure 1C). Electroconvulsion-induced DDs consisted of DLM activity of varying firing frequencies, which quickly accelerates, peaks ( $\sim 20 \mathrm{~Hz}$ ), and gradually decelerates (Figure 1C). During sustained flight, the DLM firing was more rhythmic, with highly regular spike intervals $(\sim 5-10 \mathrm{~Hz})$, whereas DLM firing during grooming differed markedly, with short bursts of spikes $\left(\right.$ ISI $^{-1}>100 \mathrm{~Hz}$ ) coincident with wing depression events.

Figure $2 \mathrm{~A}$ depicts histograms of the $\mathrm{ISI}^{-1} \mathrm{~s}$ during the respective motor programs from a representative fly. It is evident that the three motor programs had clearly distinct frequency profiles. DD seizure spiking displayed a right-skewed ISI $^{-1}$ distribution with most spiking occurring between 1 and $10 \mathrm{~Hz}$, with a clear "tail" of vanishing activity and a briefer session of fast-firing spikes, beyond $25 \mathrm{~Hz}$, corresponding with the early peak of DD firing. During flight, the distribution of $\mathrm{ISI}^{-1} \mathrm{~s}$ was markedly less variable, reflecting a rhythmic firing.
During grooming, DLM spiking displayed a highly-variable ISI $^{-1}$ distribution, with some short spike bursts exceeding 100 $\mathrm{Hz}$-well above the maximum rate observed during either DD or flight.

With an ensemble of tens of flies, we examined firing rate regularity across the population for each motor pattern, by comparing the fractional distribution of the average ISI $^{-1}$ along the spike train against that of the overall firing rate (defined as the total spike count divided by recording duration, typically $60-120 \mathrm{sec}$ ). In general, rhythmic firing produces similar results from the two measures and disparity between the two measures suggests highly arrhythmic firing. As Figure 2B illustrates, rhythmic DLM firing during flight produced highly similar distributions of firing rate based on the two measures (average $\mathrm{ISI}^{-1}=6.70 \mathrm{~Hz}$, overall firing rate $=4.90$ $\mathrm{Hz}$ ), whereas grooming behavior was associated with irregular short bursts of a few spikes during, leading to drastically different rate distributions derived from the total spike count or from the instantaneous firing frequency (average ISI $^{-1}$ vs. overall firing rate $=16.62$ vs. $0.55 \mathrm{~Hz}$ ). The discrepancy between the two measures of firing rate during delayed seizure discharges fell in between (average ISI $^{-1}$ vs. overall firing rate $=7.46$ vs. $5.08 \mathrm{~Hz}$ ). 
A
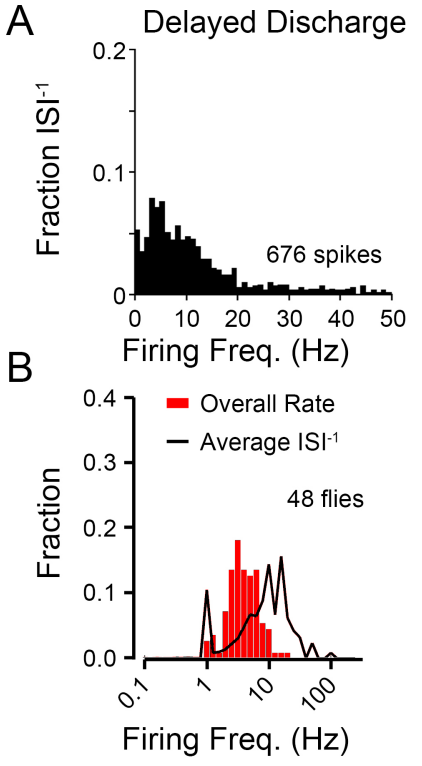

Flight

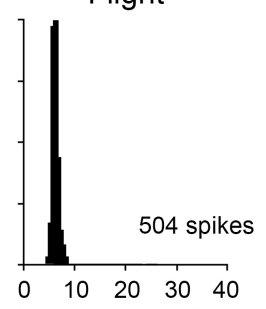

Firing Freq. $(\mathrm{Hz})$

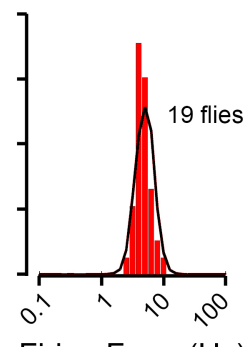

Firing Freq. $(\mathrm{Hz})$
Grooming
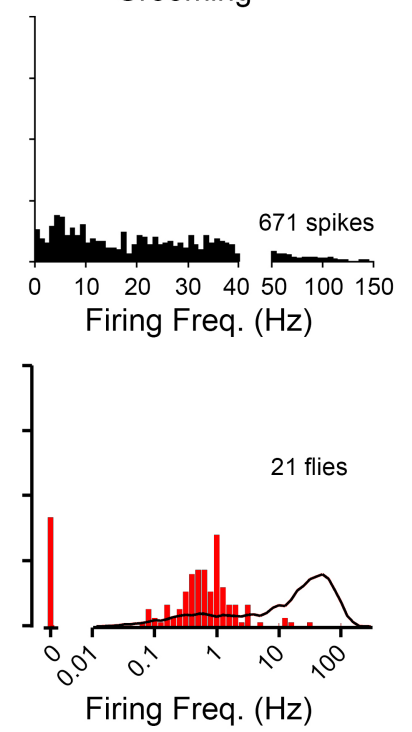

Figure 2. DLM firing characteristics during delayed seizure discharge, flight and grooming activities.

(A) Histograms of DLM instantaneous firing frequencies observed during DD, flight and grooming bouts from representative individuals. The DD histogram was constructed from nine discharges evoked in a single fly (total of $92 \mathrm{~s}$ of activity, 676 spikes), flight and grooming histograms were obtained from $130 \mathrm{~s}$ bouts of the respective behaviors (1583 and 671 spikes respectively). (B) Distributions of two measures of firing frequency during respective motor programs across a population of WT flies. Red histogram, the average of the overall firing frequency, defined as the total spike count/total recording duration for each spike train. Black line profile, the ensemble distribution of instantaneous firing frequencies $\left(\right.$ ISI $\left.^{-1}\right)$ from the same spike trains. Note that the distributions are plotted on a log-frequency scale. Sample sizes as indicated.

\section{Interactions between Bilateral DLM Motor Units dur- ing Delayed Seizure Discharges, Flight and Groom- ing}

Analysis of spike timing relationships between left and right DLM pairs can reveal potential interactions between the bilaterally symmetrical pairs of motor neurons that innervate them and can yield important clues to distinctive mechanisms of spike pattern generation among different motor programs. We recorded from top-most pair of DLMs (\#45a [47]), each of which is innervated by a contralateral MN5 motor neuron in the mesothoracic ganglion [58]. Of the three motor patterns examined here, relationships between bilateral DLM pairs during flight have been best characterized [28, 29, 53], while the relationships during grooming and delayed seizure discharges have yet to be documented. Within individual flies, we found that for flight and DD, the firing frequency profiles of the left and right DLM units were not identical, but similar to each other (Figure 3A, top two panels, data from a representative individual). In contrast, striking discrepancies in firing frequency between left-right DLM pairs were observed during grooming (Figure 3A, bottom panel). In behavioral context, the left-right pairs perform coordinately during flight and DD, but can be decoupled during grooming.

We further quantified bilateral phase relationship of DLM firing in the three motor programs by using the protocol described by Koenig \& Ikeda [53]. Briefly, the fraction of an inter-spike interval that elapsed prior to the contralateral muscle firing was measured to define the left to right (or rightto-left) phase from zero to one for individual spikes along the left (or right) DLM spike train (Figure 3B, data from a representative individual, see also Methods). Histograms of the bilateral phase relationships between contralateral units appeared relatively unremarkable during DD, compared to the other two motor programs (Figure 3C, derived from Figure 3B). An ensemble distribution created from DD spike trains across different flies did not indicate significant deviation from a uniform distribution (Figure $3 \mathrm{D}, \mathrm{p}>0.05$, $\chi^{2}$-test). However, during flight, the bilateral phase relations of DLM units displayed a characteristic spike exclusion, as indicated by the observation that immediately after a spike, the contralateral unit rarely spiked ( $\pm 10 \%$ of ISI period, approximately $40 \mathrm{~ms}$; arrows in Figure 3D). This "exclusion band" feature was previously described by Levine \& Wyman [28], and may serve to differentiate flight-associated spiking from DD seizures within individual DLMs. In contrast to the relatively uniform distributions associated with DD and bilateral exclusion during flight, grooming spike activity was characterized by phase-correlated firing in the left-right DLM pairs, with most firing occurring within $10 \%$ of the ISI period on the contralateral side. Thus, our analysis of the phase relationship provides a quantitative indicator for degrees of independence, exclusion or coupling between bilateral pairs 
A
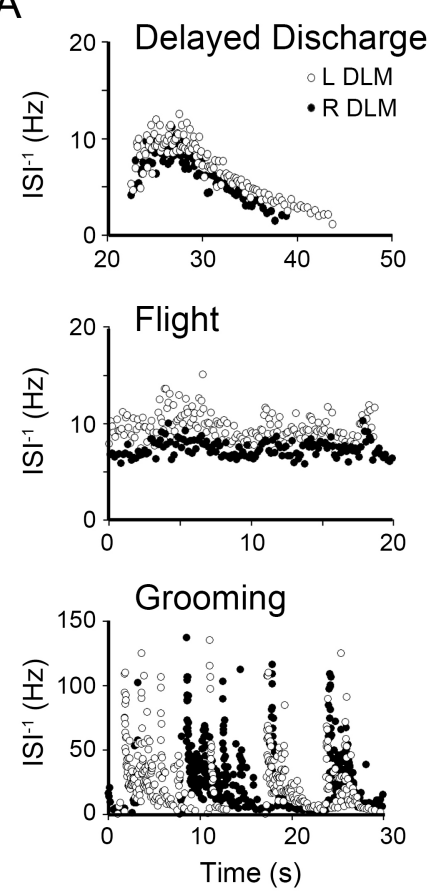

B

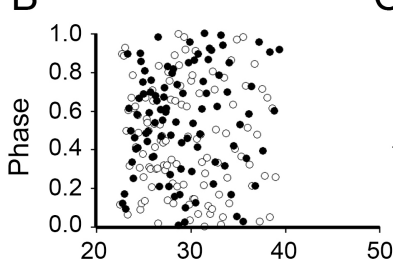

C
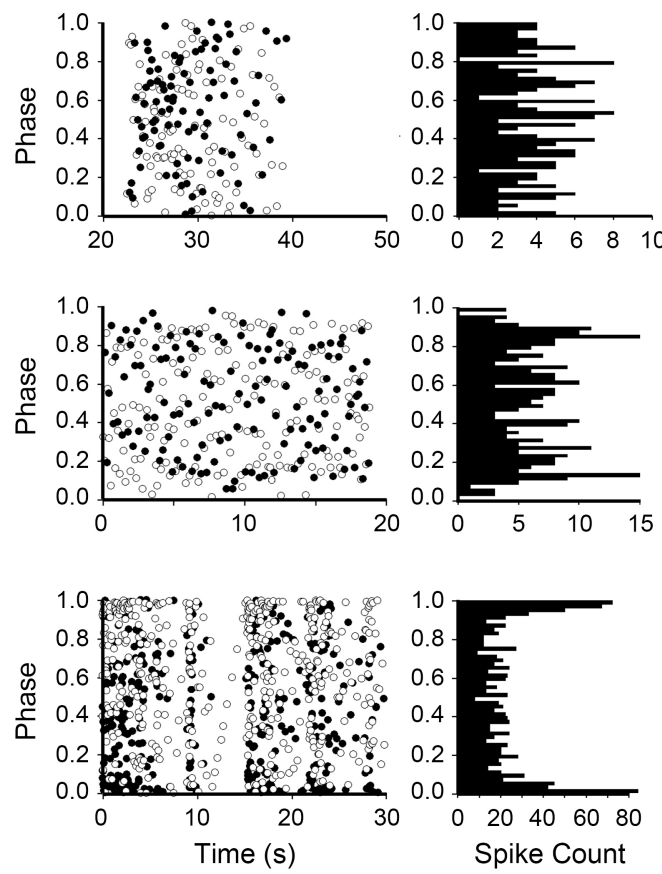
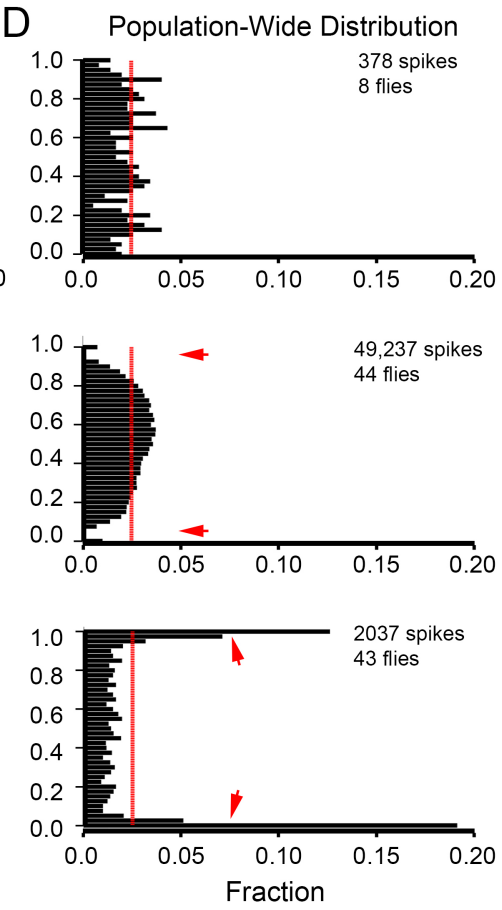

Figure 3. Timing relationships between left and right DLM spikes during delayed seizure discharges, flight and grooming. (A) Plots of the instantaneous firing frequency time course for left (open circles) and right (closed circles) DLM spiking during DD (top panel), flight (middle panel) and grooming behavior (lower panel) from a representative individual. (B) Corresponding plots of the phase relation between left and right DLM spiking for data shown in panel A. The bilateral phase relation between left and right DLM spikes was determined as the fraction within the inter-spike interval from the contralateral unit that lapsed prior to the ipsilateral spike occurrence [28,53]. Open circles indicate the phase location of the left DLM spikes with respect to the right DLM spiking, and vice-versa for closed circles. (C) Histograms of the bilateral phase relations from the right DLM spikes of the respective plots in B (closed circles). (D) Ensemble histograms of the bilateral phase relationships from the population of flies observed during the three motor programs, across the population of flies observed (sample sizes indicated). Red line indicates a uniform phase distribution. Arrows indicate phase-exclusion observed during flight and in-phase firing during grooming activity.

of DLMs during the respective motor programs.

\section{Poincaré and $\mathrm{ISI}^{-1}$ vs $\mathrm{CV}_{2}$ Plots: Distinct Signatures of Rate and Rhythmicity among DLM Spike Patterns}

In addition to the ensemble ISI $^{-1}$ distributions in a somewhat overlapping ranges (Figure 2), DD, flight and grooming spike patterns may be qualitatively distinguished based on differences in variation of ISI $^{-1}$ between adjacent spikes (Figures $1 \& 3 \mathrm{~A})$. The temporal structure of sequential spike intervals may be distinct among these firing patterns, but are not retained in the firing frequency distributions and left-right phase relationships (Figures $2 \& 3 \mathrm{~B}, \mathrm{C}$ ). Poincaré plots (also known as return maps, c.f. $[59,60]$ ) have been used to visualize spike interval dynamics, which may contain both deterministic and stochastic components. We plotted sequentially the ISI-1each of spike interval against the subsequent interval $\left(\mathrm{ISI}^{-1}{ }_{n}\right.$ vs. $\mathrm{ISI}^{-1}{ }_{n+1}$, hereafter designated as Poincaré trajectory or PT), to quantify sequential changes along the progression of a spike train (Figure 4A). When plotted in this manner, a constantfrequency spiking train will have successive points in the PT falling at the same location on the lower-left to upper right diagonal. A spike train with gradual frequency-modulation will have data points wandering along the diagonal of the plot reflecting changes in the ISI $^{-1}$. Irregular spike trains will have data points deviating from the diagonal to different degrees depending on how abruptly the sequential ISI $^{-1}$ changes. Within individual flies, grooming spike patterns displayed much higher variability in successive spike intervals in their PT, compared to both DD and flight patterns that showed lower ISI $^{-1}$ to ISI ${ }^{-1}$ variability. Thus, the PT for individual grooming spike trains traversed abruptly in broad strides across the diagonal, whereas successive data points in DD clustered closer to the diagonal and flight to almost a point (Figure 4A), uncovering the special morphological features 
A Delayed Discharge

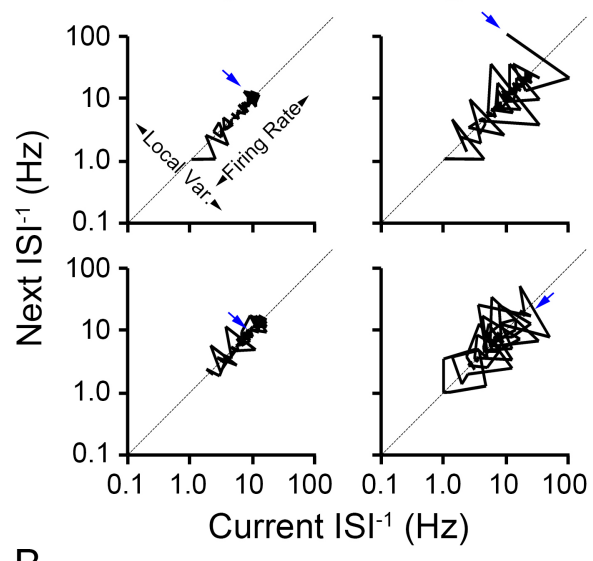

B

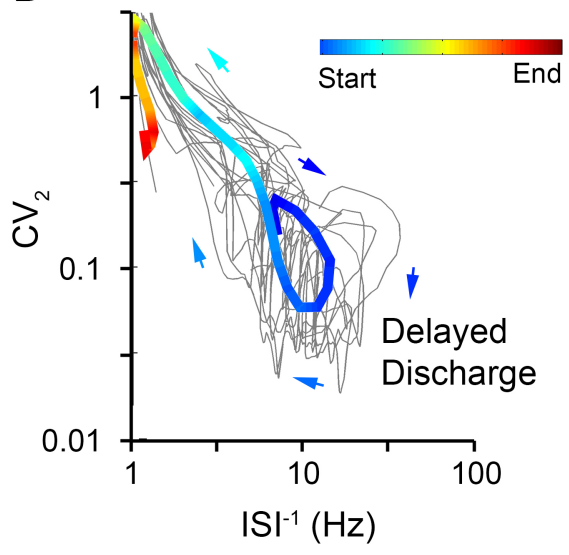

Flight
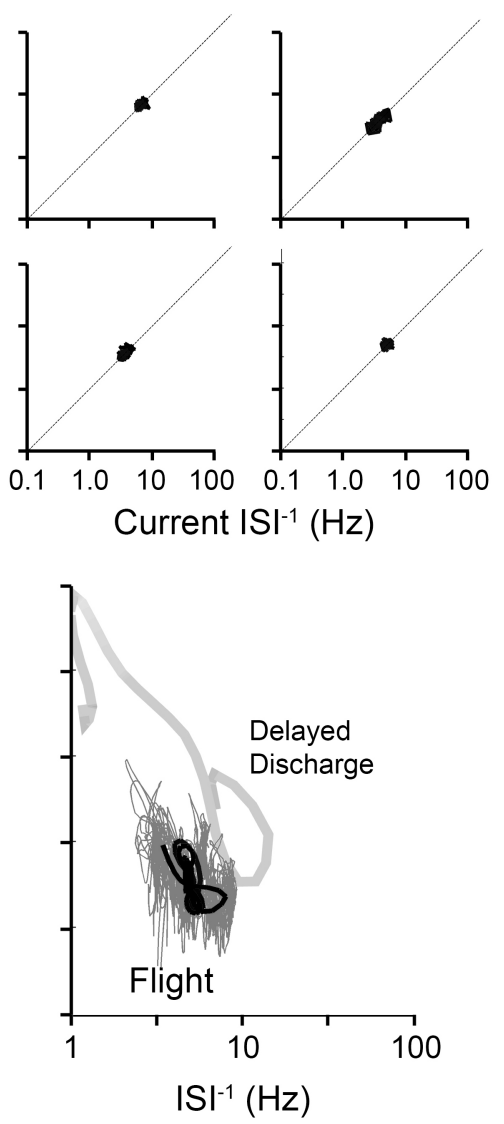

Grooming
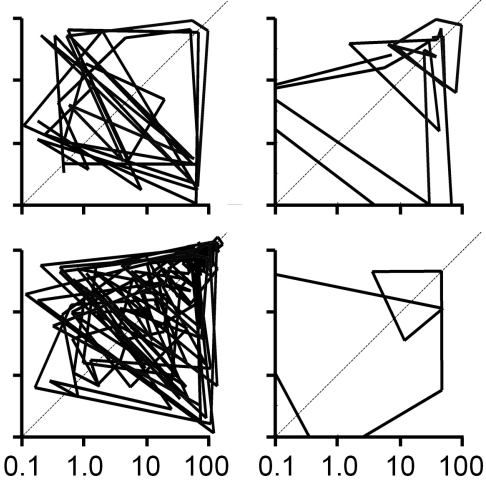

Current $\mid \mathrm{SI}^{-1}(\mathrm{~Hz})$

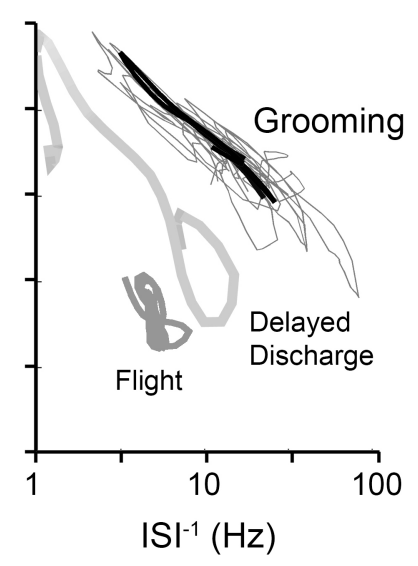

Figure 4. Poincaré plots and $\mathrm{ISI}^{-1}$ vs. $\mathrm{CV}_{2}$ trajectories of DLM spiking during delayed seizure discharges, flight and grooming. (A) Representative Poincaré plots of DD, flight and grooming spike trains. For each inter-spike interval within the spike train, the $\mathrm{ISI}^{-1}$ is plotted against the subsequent $\mathrm{ISI}^{-1}$ within the sequence. Points along the lower-left to upper-right diagonal represent stable firing frequencies while deviations from the diagonal represent degrees of change in firing frequency. Arrows indicate start of trajectories for DDs. Note that axes are plotted on a logarithmic ISI $^{-1}$ scale. Note the compact Poincaré plots of flight and the large deviations from the diagonal within the grooming plots. (B) Trajectories of the ISI $^{-1}$ versus instantaneous $^{-1}$ coefficient of variation $\left(\mathrm{CV}_{2}\right)$ for spiking during the three motor programs. Lower values of $\mathrm{CV}_{2}$ indicate more rhythmic firing. Ten sample trajectories of DD (left), flight (center) or grooming (left) trajectories are displayed (light grey), on which an ensemble average of the individual trajectories for the respective motor programs is over-laid (bold line) (ensemble average traces drawn from a sample size of 11, 126, and 115 individuals respectively). For the averaged DD trajectory, the line color and arrows indicate the trajectory's time course and the ensemble average trajectories for flight and grooming are bolded. For the flight panel, the average DD trajectory is displayed for reference (bold grey line). For grooming trajectories, the averaged DD and flight trajectories are displayed for comparison. See Methods for computation details.

of the spike trains hidden in the above population-level firing frequency distributions (Figure 2B).

The above Poincaré trajectories clearly illustrated distinctions among the three motor patterns. However, multiple PTs cannot be readily treated to produce an ensemble trajectory based on larger sample sizes to highlight the global dynamic differences between motor patterns. We employed a transformation of the PTs to enable over-lays of individual trajectories for construction of an 'average' trajectory across a number of individual trajectories (Figure 4B). It is known that the distributions of both instantaneous firing frequency and variability between successive spike intervals can be extracted directly from the Poincaré plot $[52,61]$. Since deviations of the PT from the diagonal are related to variability of spike train intervals, one established treatment is to quantify the deviation from the diagonal along the local firing frequency adjacent to each spike in the sequence by adopting a measure for the instantaneous coefficient of variation $\left(\mathrm{CV}_{2}\right)$, defined as $2\left|\mathrm{ISI}_{n}^{-1}{ }_{n}-\mathrm{ISI}^{-1}{ }_{n+1}\right| /\left(\mathrm{ISI}^{-1}{ }_{n}+\mathrm{ISI}^{-1}{ }_{n+1}\right)([52]$ see also Methods). The transformed plot of the $\mathrm{ISI}^{-1} \mathrm{vs} \mathrm{CV}_{2}$, using the 
same sequential ISI $^{-1}$ information, is essentially a re-scaling of the sequential deviations from the diagonal in the PT plotted against the $\mathrm{ISI}^{-1}$. After this transformation, high $\mathrm{CV}_{2}$ values correspond with abrupt, large changes in adjacent spike intervals in either direction, increasing or decreasing.

This treatment generates $\mathrm{ISI}^{-1}$ vs $\mathrm{CV}_{2}$ plots that display clear distinctions among the three motor programs with an advantage of generating ensemble statistics for repeated trials (see Methods for averaging details). During delayed seizure discharges (DD), average trajectory of $\mathrm{ISI}^{-1}$ vs $\mathrm{CV}_{2}$ (Figure 4B, left panel, temporally color coded, average trajectory superimposed on individual trials in grey lines) illustrates a stereotypic trend of an initial acceleration (ISI ${ }^{-1}$ from $\sim 7$ to $\sim 15 \mathrm{~Hz}$, blue segments) accompanied by increasing regularity, lower $\mathrm{CV}_{2}(<0.1)$, which then gradually decreases in firing frequency as well as regularity with increasing $\mathrm{CV}_{2}$ values (Figure 4B, left panel, cyan-green segment, climbing towards the upper-left corner, cf. Figure 11C, left panel). For sustained flight, DLM spiking appears as a tight trajectory with relatively little drift within a confined region (Figure 4, middle panel, ISI $^{-1} \sim 5 \mathrm{~Hz}, \mathrm{CV}_{2} \sim 0.03$, cf. Figure $1 \mathrm{C}$ middle panel). Notably, the initial phase of the DD trajectory and the flight trajectories occupy adjacent regions of low $\mathrm{CV}_{2}$ values; although for DD (thick grey average trajectory re-plotted in Figure 4B for comparison), instantaneous firing frequencies are somewhat higher $(\sim 10-15 \mathrm{~Hz}$ vs $3-10 \mathrm{~Hz})$ across the population sampled. In contrast, for the case of grooming, DLM spike trajectories occupy a region separate from either $\mathrm{DD}$ or flight, displaying significantly higher $\mathrm{CV}_{2}$ values, usually above 0.5 , consistent with the qualitative observations of arrhythmic firing punctuated with abrupt changes in ISI $^{-1}$ (Figure 4B, right panel cf. Figure 1C right panel). Thus, the temporal evolution of the spike patterns of the three motor programs are intrinsically distinct since the above quantitative treatment generates averaged trajectories that occupy three separable regions in the $\mathrm{ISI}^{-1}$ vs. $\mathrm{CV}_{2}$ plot.

\section{Differential Vulnerability of Three Motor Patterns to Genetic Perturbations of Synaptic Transmission Sys- tems}

To provide a first glimpse of the general vulnerability of spike patterns of the three motor programs to perturbations of the major synaptic transmission systems, we examined mutants of three genes that have been well-established to regulate inhibitory, excitatory and electrical synapses in the CNS: resistant to dieldrin ( $R d l$, encoding the inhibitory $\mathrm{GABA}_{\mathrm{A}}$ receptor subunit [39]), choline acetyltransferase (Cha, acetylcholine synthesis enzyme [40]) and ShakingB (ShakB, encoding innexin gap junction subunits[62, 63, 64]). These mutants have been well-characterized for their effects on the giant-fiber pathway, from the brain afferents to DLM output $[41,42,21]$. We specifically examined the $R d l^{M D-R R}$ allele, a natural variant of $\mathrm{Rdl}$ that has developed resistance to the insectide dieldrin, which also displays enhanced GABAergic inhibition $[65,66,67]$. The $C h a$ allele examined, $C h a^{t s 2}$ dis- plays defects in cholinergic transmission at room temperature, even though its enzymatic activity is more severely disrupted at elevated temperatures [40, 41]. Both $S h a k B$ alleles studied here (ShakB ${ }^{2}$ and $S h a k B^{\text {Pas }}$ ) are known to severely compromise signal transmission along the GF pathway that involves identified electrical synapses [42, 68].

We first examined instantaneous firing frequency of the three motor patterns (cf. Figure 1). Notably, DD discharges in the respective mutants showed distinct defects (Figure 55A). A substantial reduction was observed in both the number of spikes and the peak instantaneous firing frequency in $R d l \mathrm{mu}$ tants with enhanced $\mathrm{GABA}_{\mathrm{A}}$ receptor function (mean peak ISI $^{-1}$ of 6.4 vs $13.0 \mathrm{~Hz}$ for WT, Figure 5Aii). Interestingly, disruption of excitatory cholinergic transmission in Cha mutants also led to a reduction in instantaneous firing frequency $\left(\mathrm{ISI}^{-1}, 6.3 \mathrm{~Hz}\right.$ ), comparable to $R d l$ mutants. In contrast, altered electrical transmission in ShakB mutants did not significantly alter the peak ISI $^{-1}$ in the DD (Figure 5Aii). However, the qualitative temporal profile was more resistant to these genetic manipulations, since across the three mutants, the rate of rise to the peak firing frequency remained unaltered, despite the clear modifications in their firing frequencies, (Time to Peak, Figure 5Aiii).

Compared to the clear effects on DD spike trains, the flight and grooming pattern generators showed much higher resilience to the same perturbations of the above transmission systems. During flight, only $R d l$ mutants displayed a modest reduction in firing frequency compared to WT counterparts (mean $\mathrm{ISI}^{-1}$ of $5.9 \mathrm{~Hz}$ vs $8.2 \mathrm{~Hz}$ respectively, Figure 5B). For grooming spike patterns, which is intrinsically highly variable, we did not detect significant differences among the mutants tested (Figure 5C), with average mean $\mathrm{ISI}^{-1}$ ranging from 0.39 to $1.22 \mathrm{~Hz}$ across genotypes, and maximum ISI $^{-1}$ observed between 92 and $120 \mathrm{~Hz}$.

Given the clear alterations in ISI $^{-1}$ profiles of DD spike trains compared to the other motor patterns, we further characterized the impacts of $R d l$, Cha and ShakB mutations on other parameters of ECS discharge repertoire (Figure 6A, cf. [22]). Specifically we examined the stimulation threshold to trigger $\mathrm{DD}$, the onset timing of $\mathrm{DD}$, its duration, and the duration of transmission failure along the GF pathway following electroconvulsive stimulation. While WT and $R d l$ displayed similar thresholds and DD durations (Figure 6B-C), Cha and ShakB were apparently hypoexcitable as indicated by significantly higher ECS thresholds, and the reduced DD duration (mean durations of 10.0 and $9.4 \mathrm{~s}$ respectively) than WT and $R d l$ (13.3 and 11.8 s, Figure 6C). Notably, only Cha affected the onset time of DD (22.7 vs $18.3 \mathrm{~s}$ for WT, Figure 6D), which has been seen only in a subset of hyperexcitability mutants [22]. In contrast, all three mutants examined displayed prolonged periods of GF failure upon electroconvulsive stimulation, as determined by DLM failure to respond to $1-\mathrm{Hz}$ brain stimuli (Figure 6A, cf. [22]). Modest increases in DLM failure was observed in $R d l$ and $C h a$ (36.8 and 36.0 vs $26.0 \mathrm{~s}$ for WT) and extreme lengthening in ShakB (>80 s, Figure 

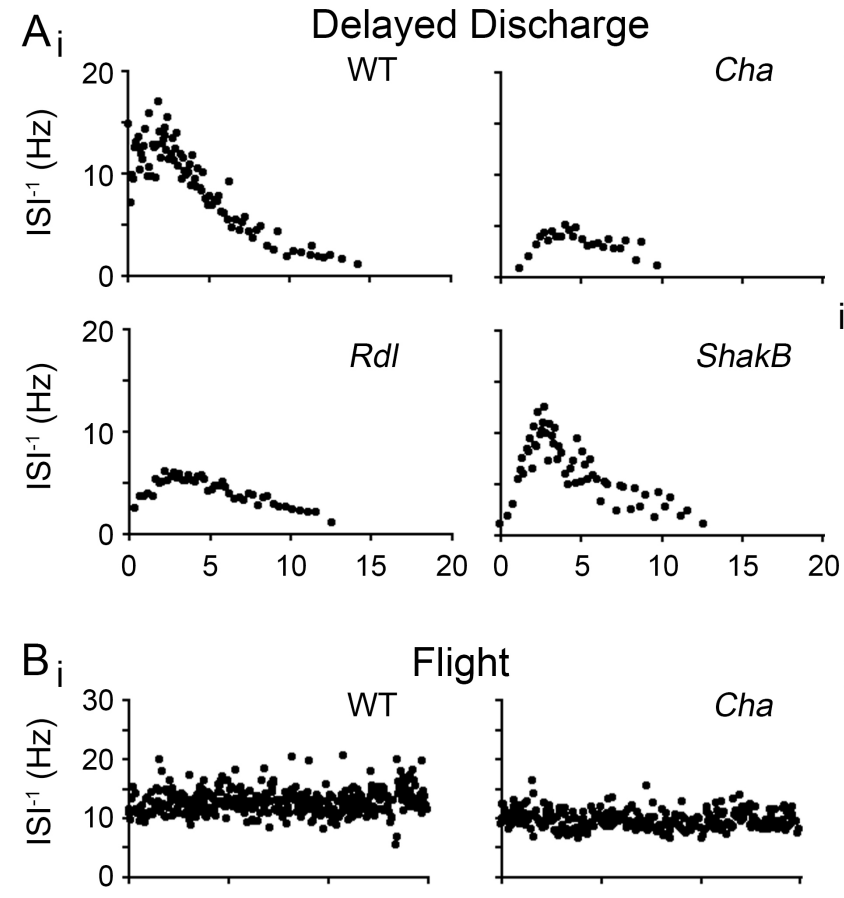

Flight
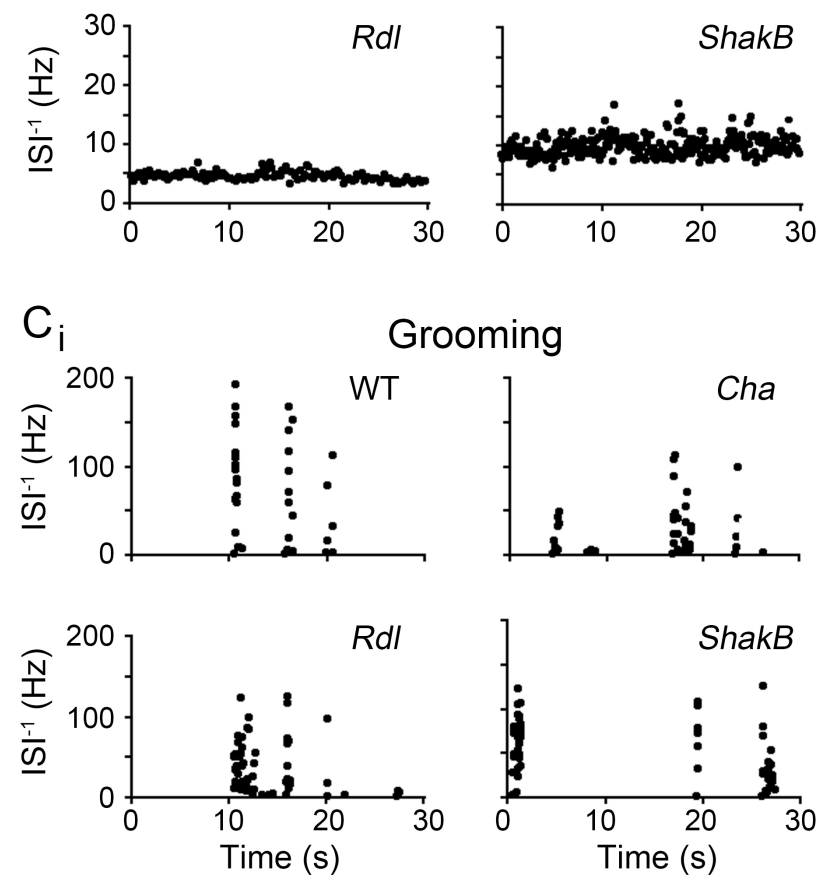

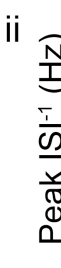
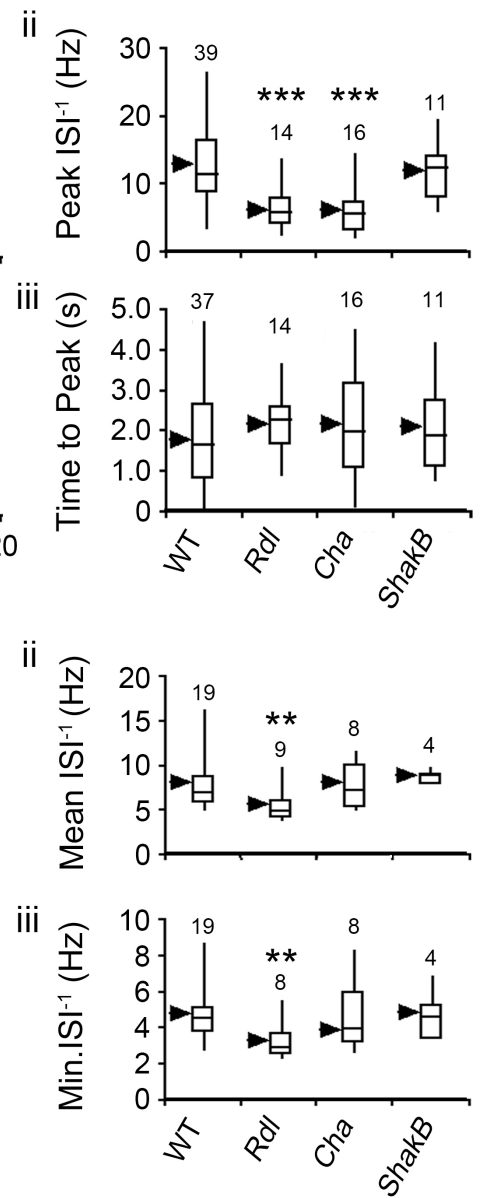

ii
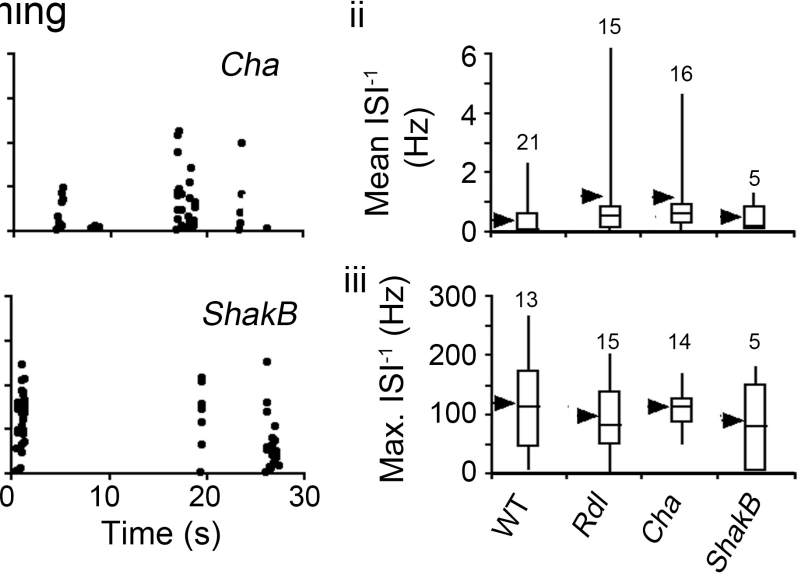

Figure 5. Modifications of DLM firing characteristics during DD, flight and grooming activity in WT, and the mutants of $\mathrm{GABA}_{\mathrm{A}}$ receptor, $R d l$, choline acetyltransferase, Cha, and electrical synapse, ShakB.

Instantaneous firing frequency (left) and sample population statistics (right) in indicated genotypes. (Ai) Representative instantaneous firing frequency plots during DD. (Aii) Box plots of peak instantaneous firing frequency. (Aiii) Time to peak frequency in the respective mutants. (Bi) Sample plots of instantaneous firing frequency during flight in the respective mutants. (Bii) Box plots of mean instantaneous firing frequency. (Biii) Minimum frequency for the respective synaptic transmission mutants. (Ci) Sample plot of instantaneous firing frequency during grooming in the respective mutants. (Cii) Mean and (Ciii) Maximum instantaneous firing frequencies during grooming. Boxes represents the $25^{\text {th }}, 50^{\text {th }}$, and $75^{\text {th }}$ percentiles while whiskers indicate $5^{\text {th }}$ and $95^{\text {th }}$ percentiles of the distribution. Arrowheads indicate distribution mean. Sample sizes as indicated. $(* * \mathrm{p}<0.01, * * * \mathrm{p}<0.001$, Mutant vs. WT, Student's T-test $)$ 
bioRxiv preprint doi: https://doi.org/10.1101/481234; this version posted January 22, 2019. The copyright holder for this preprint (which was not certified by peer review) is the author/funder, who has granted bioRxiv a license to display the preprint in perpetuity. It is made available under aCC-BY-NC 4.0 International license.

Seizure Discharge Spike Patterning in Drosophila - 10/19

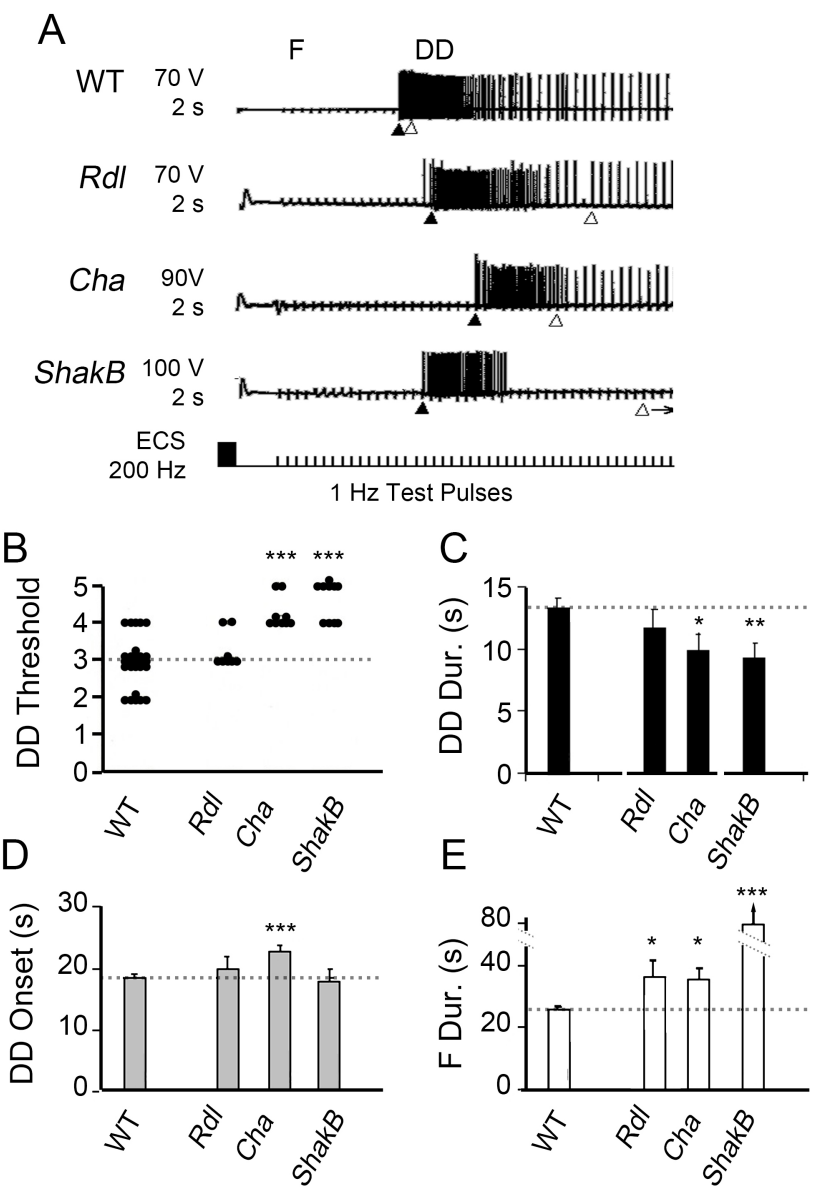

Figure 6. Electroconvulsive stimulation-evoked seizures in WT and $C h a, R d l$, and $S h a k B$ mutants.

(A) Representative traces of electrical stimulation-evoked GF failure (F) followed by DD in the respective mutants. The onsets of DD (closed triangle) and recovery of GF transmission (open triangle) are indicated below each trace. The stimulation intensity and duration (indicated) in these sample traces were significantly higher than the threshold to trigger DD patterns at saturation levels (Lee \& Wu, 2002).

(B) Threshold of stimulation required to trigger DD in respective mutants. Increments in values correspond to approximate doubling ECS intensity $(1: 50 \mathrm{~V}, 0.5 \mathrm{~s} ; 2: 50 \mathrm{~V}$, $1 \mathrm{~s}, 3: 100 \mathrm{~V} 1 \mathrm{~s} ; 4: 100 \mathrm{~V}, 2 \mathrm{~s} ; 5: 100 \mathrm{~V}, 4 \mathrm{~s}$; cf. Lee \& Wu 2006). (C) Duration of DD evoked in respective mutants. (D) Elapsed time between ECS and DD onset. (E) Elapsed time between ECS and GF transmission recovery from failure (F Duration) in mutants examined. Note that in ShakB GF transmission often failed throughout the entire recording session (lasting $>80 \mathrm{~s}$ ). For B-E $* \mathrm{p}<0.05, * * \mathrm{p}<0.01$, $* * * \mathrm{p}<0.001$, Mutant vs WT values, Student's T-test.

$6 \mathrm{E})$, a powerful demonstration for the important role of electric synapses in the transmission along the GF pathway that mediate the escape reflex $[42,34,33,68]$.

\section{Poincaré Plots and $\mathrm{CV}_{2}-\mathrm{ISI}^{-1}$ Maps as Distinct Signa- tures for Electoconvulsive Seizure Spike Patterning in Synaptic Transmission Mutations}

The diverse range of alterations to delayed seizure discharges evident in the above analyses (Figures 5 \& 6) is corroborated by characteristic differences further revealed through Poincaré plots and $\mathrm{ISI}^{-1}-\mathrm{CV}_{2}$ trajectories of DD spike trains (cf. Figure 4) in neurotransmission system mutants (Figure 7). We found the reduction in peak firing rate of DD in $R d l$ mutants (Figure 6A) was accompanied by an increase in irregular firing, indicated by relatively large deviations from the lower-left to upper-right diagonal of Poincaré plot (Figure 7A). The same spike trains plotted on $\mathrm{ISI}^{-1}-\mathrm{CV}_{2}$ phase trajectory (cf. Figure 4B) also illustrate more variable trajectories, lacking a general characteristic trajectory compared to WT (Figure 7B). In contrast, Cha mutants showed somewhat less irregular firing and $\mathrm{ISI}^{-1}$ vs $\mathrm{CV}_{2}$ trajectories. However, both $R d l$ and $C h a$ DD trajectories appeared to be confined to region with a modest "left-shift" compared to WT, consistent with decreased $\mathrm{ISI}^{-1}$ values described above (cf. Figure 5A). Although ShakB displayed a similar peak ISI $^{-1}$ value compared to WT flies (Figure 5A), the Poincaré plot indicates more erratic variability in firing frequency within DDs (Figure 7A), which is also illustrated in the ISI $^{-1}$ vs $\mathrm{CV}_{2}$ trajectories, with many trajectories scattered over a wide range (Figure 7B). However, the average trajectory suggests similar firing frequency range even though ShakB displayed shorter trajectories compared to WT (Figure 7B).

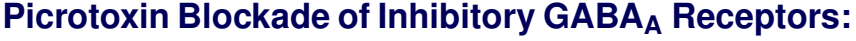 Evolving Seizure Activity Patterns from Flight-Like Firing to Bursting Discharges}

The enhanced $\mathrm{GABA}_{\mathrm{A}}$ receptor function by the $R d l^{M D-R R} \mathrm{mu}-$ tation reduced firing rate and altered DLM spike patterning without detectable changes in ECS threshold (Figures 5 \& 6). We examined the effects of suppressing the GABAergic system on motor patterns, including seizure-like behaviors. Ingestion of picrotoxin (PTX), a non-competitive antagonist of ionotropic $\mathrm{GABA}_{\mathrm{A}}$ receptors [69] results in spontaneous spasms that are accompanied by DLM spike discharges [21]. We extended this previous observation to carry out quantitative comparisons between PTX- and ECS-induced discharges, DD. We found that application of a small drop of PTX solution $(1 \mathrm{mM}, \sim 0.1 \mu \mathrm{l})$ to an incision on the compound eye resulted in abnormal DLM spiking, starting within 10 min of application (Figure 8A). Initially, DLM firing appeared rhythmic, with spiking frequencies somewhat higher than those observed during tethered flight (mean ISI ${ }^{-1} \sim 10-20 \mathrm{~Hz}$, Figure 8B, cf. Figure 1B). Over the course of 30 minutes, short bursts of spikes emerged (Figure 8A) consistent with the previous report on PTX-fed flies [21]. The ISI ${ }^{-1}$ of spikes within these bursts sometimes exceeded $100 \mathrm{~Hz}$ (Figure 8B), and the inter-spike intervals were highly variable. These two modes of PTX-induced spiking activity are referred to as 'flightlike' and 'bursting' activity respectively below. Importantly, 

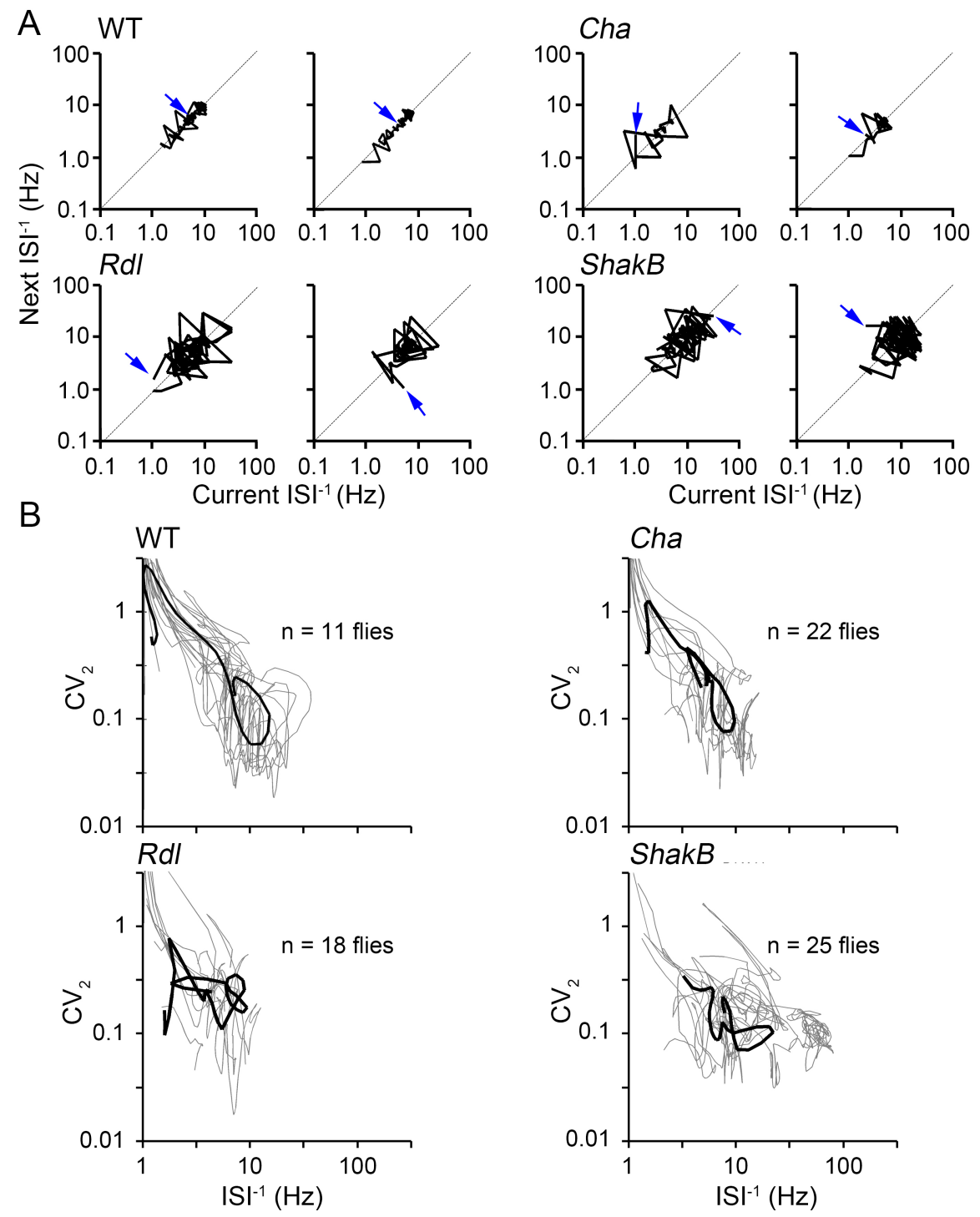

Figure 7. Poincaré plots and $\mathrm{ISI}^{-1}$ versus $\mathrm{CV}_{2}$ trajectories of DD in synaptic transmission system mutants.

(A) Representative Poincaré plots of DD spike trains in WT, Rdl, Cha and ShakB mutants. Arrow indicates initial inter-spike interval of the spike train. (B) $\mathrm{ISI}^{-1}$ versus $\mathrm{CV}_{2}$ trajectories from DDs in 10 representative individuals (grey lines) from the sample population and averaged trajectory (dark bold line) of DDs across the entire sample population from each genotype (sample size as indicated).

the sequence of PTX-induced DLM spiking was gradual and continuous. Furthermore, distinct from ECS-evoked seizure pattern, a period of paralysis followed by a stereotypic discharge pattern was missing in PTX-induced motor activity, suggesting two different modes of seizure activity.

An analysis of the phase relations between bilateral pairs of DLM units revealed striking distinctions between the flightlike and bursting spike patterns induced by PTX (Figure 8CD). Flight-like firing displayed a relatively uniform phase distribution, with a clear spike-exclusion band approximately $\pm 10 \%$ of contralateral spike (Figure $8 \mathrm{D}$ ), resembling the observations during sustained flight (Figure 3D). Bursting spik- ing activity, in contrast, displayed a phase-relation histogram with most firing occurring in-phase, resembling the bilateral relationships observed during grooming (Figure 8D, cf. Figure 3D).

In addition to the application of PTX via an incision in the compound eye, we adopted a second procedure, dorsal vessel injection (DV injection, [48]) to investigate whether a global, systemic application of PTX could induce additional spiking patterns. The dorsal vessel serves as a major pulsatile organ which circulates hemolymph [70], and injected solutions circulate throughout the fly within a few seconds. We injected a fixed volume of solution marked with blue \#1 dye ( $0.33 \mathrm{ul})$ in 

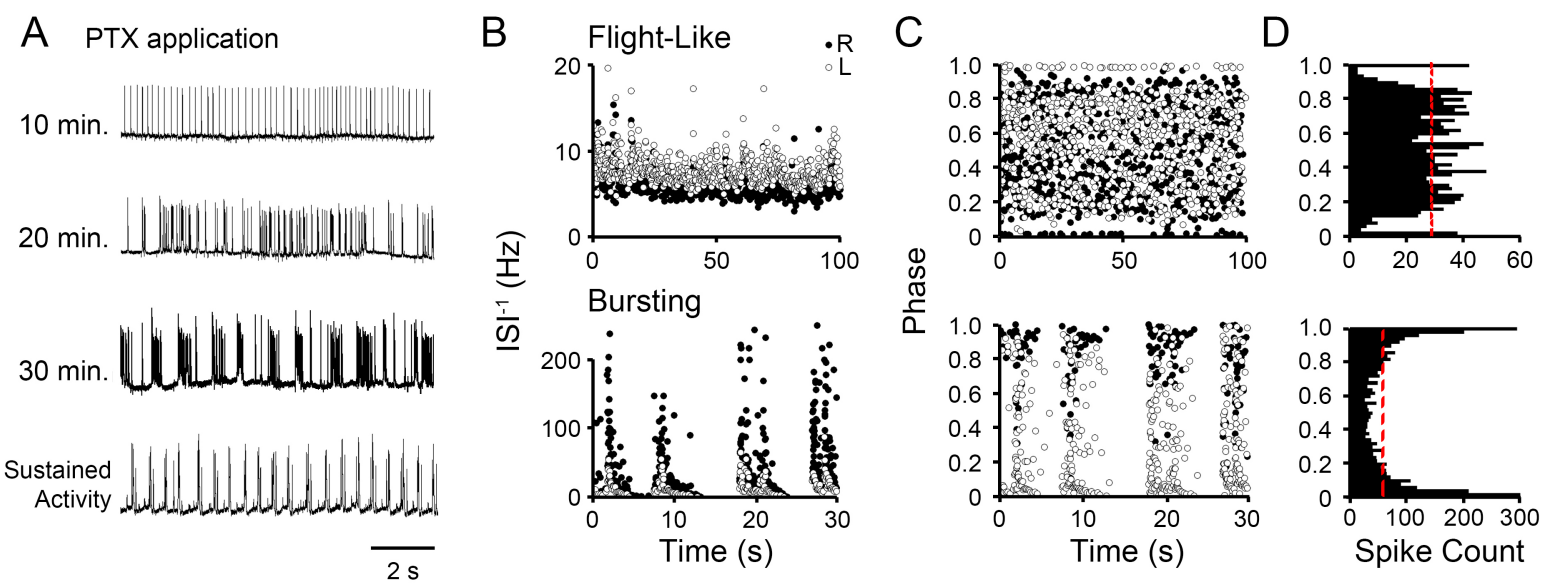

Figure 8. DLM spiking patterns evoked by picrotoxin application.

(A) Representative traces of DLM spikes triggered by PTX applied to a punctured eye (see Methods for details) at $10 \mathrm{~min}$ intervals over $40 \mathrm{~min}$. Note the transition from rhythmic "flight-like" pattern to repetitive burst discharges. (B) Plots of ISI ${ }^{-1}$ during flight-like and bursting periods from a single individual. Spiking in the left and right DLMs is indicated by open and closed circles respectively. (C) Corresponding phase relations between left and right DLM spiking during the flight-like and bursting states shown in B. (D) Histogram of bilateral phase relations in (C). Note the similarity between the PTX-evoked flight-like and burst firing with respectively the phase relations during flight and grooming shown in Figure 3C-D.

the tethered fly (Figure 9A, Supplemental Video 2) to ensure the systemic spread of PTX throughout the abdomen thorax and head. We found the two approaches induced the same sequence of spike patterns even though DV injection triggered these events on an accelerated timescale. As Figure 9A shows, PTX injection led to a stereotypic repertoire of a brief wing buzz followed by a 'wings-up' posture (see also Supplemental Video 2). Consistent with eye application, PTX injection between 50 and $100 \mu \mathrm{M}$ induced flight-like DLM spike patterns which evolved into bursting activities within seconds to minutes (Figure 9B). At concentrations of $200 \mu \mathrm{M}$ or above, we found the flight-like firing pattern was skipped and progression of burst discharge patterns was observed within seconds of injection (Figure 9B).

The two categories of PTX-induced spike patterns are clearly contrasted by their trajectories in Poincaré plots (Figure 9C). The flight-like spike patterns displayed PTs which were relatively compact, with occasionally sharp deviations, corresponding to spike doublets or brief gaps in firing, which were notably absent during sustained flight in wild-type flies (compare to Figure 4A). Bursting spike patterns displayed strikingly different PTs, characterized by stereotypic recurrent sequences of successive ISI $^{-1}$ values, corresponding to recursive bursts. Plots of the $\mathrm{ISI}^{-1}$ vs. $\mathrm{CV}_{2}$ served to further contrast these features (Figure 9D). PTX-induced flight-like firing displaying a more stable phase trajectory with $\mathrm{CV}_{2}$ $\sim 0.1$, and ISI ${ }^{-1}$ higher than flight $(\sim 10-20 \mathrm{~Hz})$, whereas bursting appeared as a looping trajectory. Each burst cycle started with at a relatively lower ISI ${ }^{-1}$ and high $\mathrm{CV}_{2}$, then accelerated to high $\mathrm{ISI}^{-1}$ and lower $\mathrm{CV}_{2}$, corresponding to the long inter-burst interval followed by the fast peaking and slow decay of the firing frequency within the burst.

\section{Discussion}

Due to the ease of electrical monitoring, coupled with the tractable genetic manipulations in Drosophila, the indirect flight muscles, DLMs, have been frequently used for studying consequences of mutations of identified genes on membrane excitability and synaptic transmission (for early examples, see $[71,19,72,73]$. Mutational analyses of a number of motor programs that drive DLM activity have also been carried out. DLM responses have enabled characterization of the GF pathway-mediated jump-and-flight escape reflex triggered by visual or vibrational stimulation $[41,34,35,74,68$, $75,76,77]$ which leads to escape-related wing depression $[33,78]$. Additionally, DLM recording has enabled detailed genetic analyses of the pattern generators that drive flight [79, 80, 27], grooming [34, 81, 57], and electroconvulsively induced seizure discharges, including the initial discharge, ID, and delayed discharge, DD have also been carried out [20, 82, 23, 21, 22, 83].

In the present study, direct comparisons of spike patterning aim at an initial qualitative and quantitative delineations among three distinct modes of DLM spiking associated with $\mathrm{DD}$, flight, and grooming motor programs.

\section{DLM-Activity Signatures Associated with Seizures, Flight and Grooming Motor Patterns}

The spiking activity of the six DLMs on each side of the fly thorax is driven by five motor neurons in the mesothoracic ganglion, with the lower four muscles (DLMc-f) each innervated by a single motor neuron (MN1 - 4) on the ipsilateral side, while the top two muscles (DLMa and DLMb) are innervated by a single contralateral motor neuron, MN5 $[58,84,85]$. The MN5 is particularly well characterized, in terms of neurite 

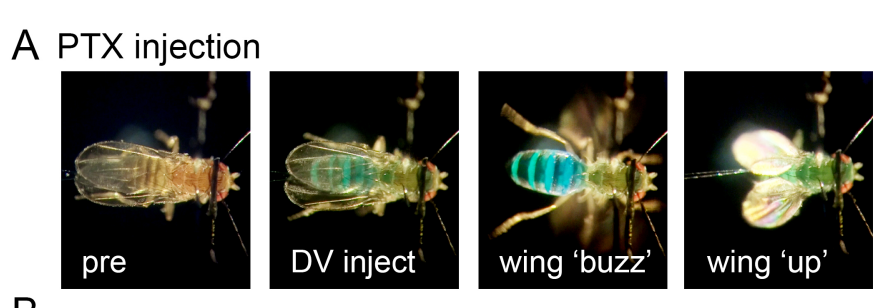

B
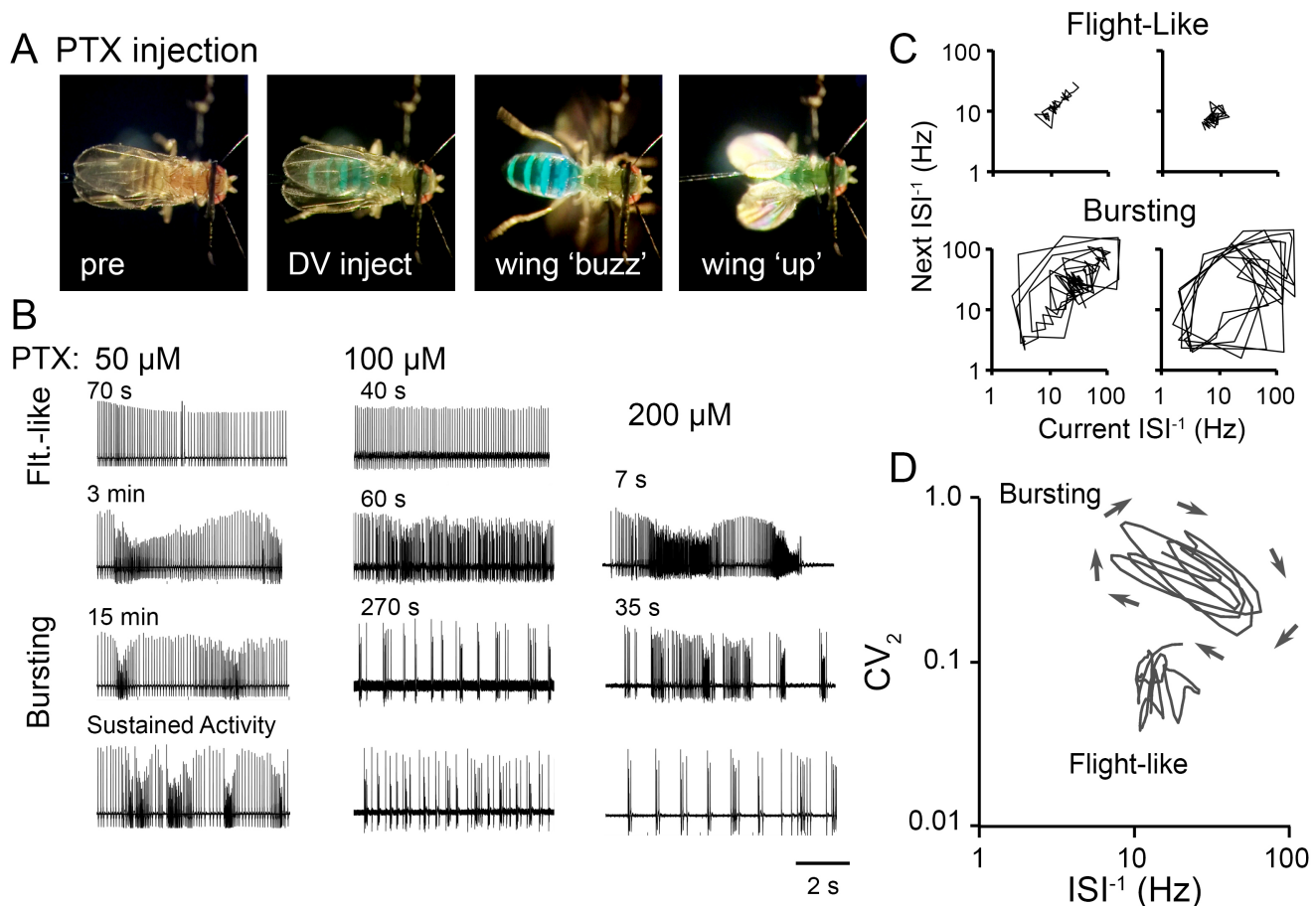

Figure 9. Rapid effects from systemic dorsal vessel (DV) injection of picrotoxin.

(A) Video frames illustrating behavior and posture from a fly before and after $100 \mu \mathrm{M}$ PTX DV injection. A transient wing 'buzz' was often observed, and flies displayed a 'wings-up' posture for several hours before death. (B) Representative spiking traces from three individual flies injected with either 50, 100 or $200 \mu \mathrm{M}$ PTX, respectively. Traces illustrate the sequential events, evolving from flight-like to bursting patterns, at different times after injection as indicated. At $50 \mu \mathrm{M}$, discrete inter-burst gaps did not fully develop, and at $200 \mu \mathrm{M}$, the initial flight-like state was not captured and spike activity (7 s) rapidly evolved into bursting activity. (C) Poincaré plots of DLM spiking displaying flight-like or bursting spike patterns $(\sim 5$-s) from two representative individuals (100 $\mu \mathrm{M}$ PTX inject). (D) $\mathrm{ISI}^{-1} \mathrm{vs} \mathrm{CV}_{2}$ trajectories from $\sim 5$-s portions of the spike trains shown in $\mathrm{C}$ during the flight-like and bursting DLM spiking phases.

outgrowth $[86,87,88]$ as well as excitability [89]. Individual $\mathrm{Ca}^{2+}[90]$ and $\mathrm{K}^{+}$[91] currents within the MN5 soma have been characterized, and their contributions to action potential generation computationally modeled [92]. Notably, the MN5 and the DLMa-b fibers are capable of following direct stimulation one-to-one well beyond $100 \mathrm{~Hz}[73,34]$. Thus, recording from DLMa or DLMb faithfully registers the MN5 activity, facilitating the analyses of a variety of motor patterns spanning a wide range of firing frequencies.

To quantitatively differentiate DLM spiking patterns associated seizure discharges, flight and grooming activities (Figure 1), we characterized several parameters to capture salient features of spike firing frequency (Figure 2), spike timing relationships between contralateral units (Figure 3), and the evolving trajectory of varying firing regularity and frequency during episodes of spiking activity (Figure 4). The instantaneous firing frequency, ISI $^{-1}$, has been extensively utilized to quantify DLM spiking frequency (e.g. [93, 53]), and our analysis revealed distinctive ISI $^{-1}$ distributions during the three respective motor programs. Further, the spike timing relationships between the left and right units (Figure 3 ), as well as temporal characteristics revealed by Poincaré plots and $\mathrm{ISI}^{-1}-\mathrm{CV}_{2}$ trajectories clearly separate these firing patterns into distinct categories (Figure 4).

Consistent with previous reports $[29,53]$, we noted that the left-right phase distribution of flight spike trains displayed "exclusion band" in which synchronous firing between the bilateral DLM pair is very rare. In contrast, during DD spiking no discernable phase relation in timing between the left and right units was observed, while grooming spikes tended to be phase-correlated (Figure 3 ). These findings suggest that the exclusion band is specific to flight motor patterns, although the precise cellular and molecular mechanisms remain to be uncovered. Indeed, electrical coupling between motor neurons [53], or inhibitory glutamate channels (gluCl[94]) have been proposed to mediate this mutual feedback inhibition.

Among DD, flight and grooming, a readily apparent qualitative distinction is the ISI variation in their spike patterns, with low variability associated with flight, high variability with grooming, and DD in between (Figure 1). To contrast the spike interval variability associated with the three motor patterns, we employed two related approaches to analyze spike trains, Poincaré plots (Figure 4A) and of the $\mathrm{ISI}^{-1} \mathrm{vs} \mathrm{CV}_{2}$ plots (Figure 4B). Poincaré plots have been frequently utilized in characterizing variability in heart beats (e.g. $[95,96])$ and in neuronal action potentials (e.g. [97]). We found that Poincaré 

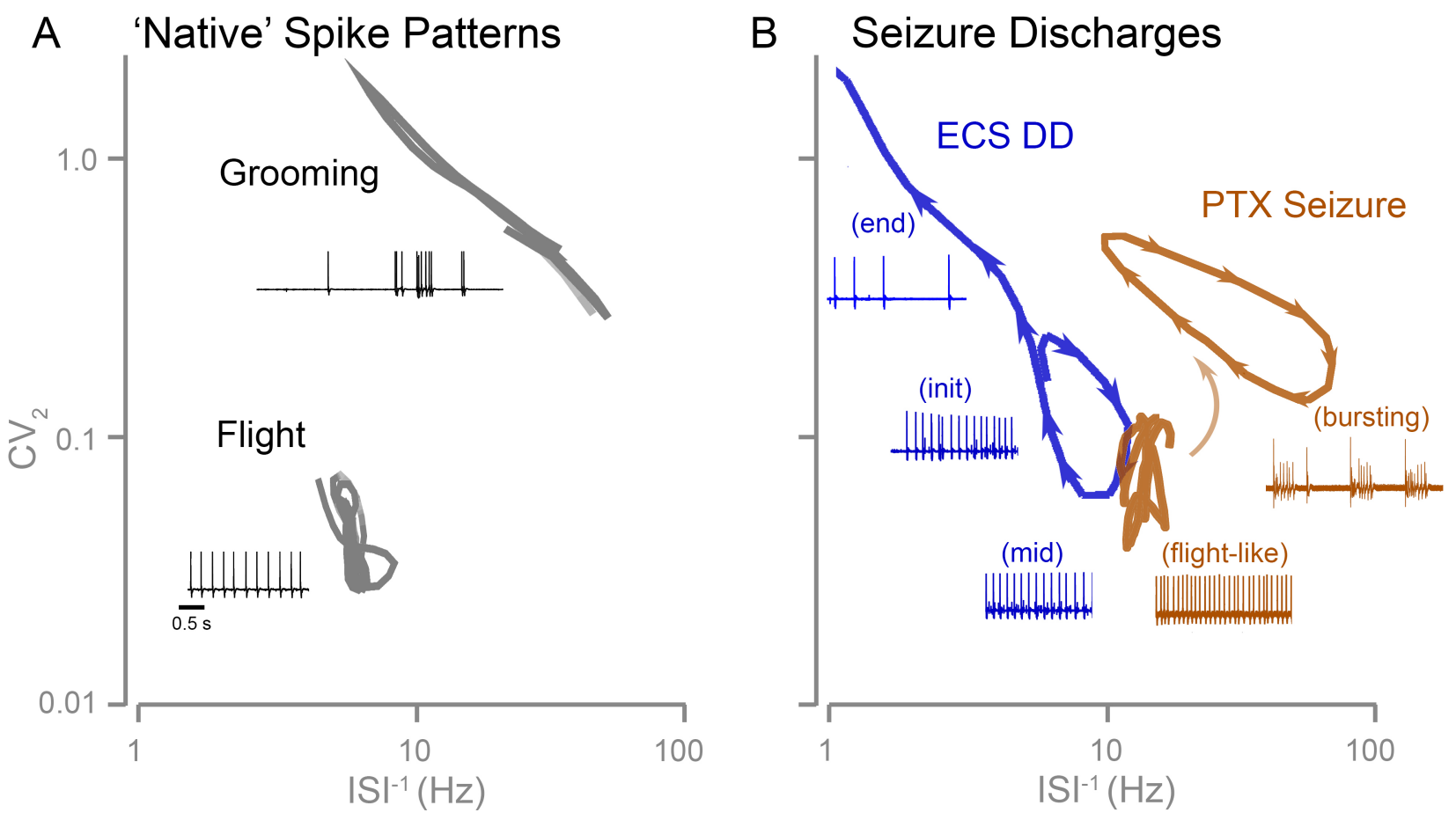

Figure 10. ISI ${ }^{-1}$ vs $\mathrm{CV}_{2}$ plots reveal distinctions in DLM spike patterns during flight, grooming and seizure discharges. Summary of averaged $\mathrm{ISI}^{-1}$ vs $\mathrm{CV}_{2}$ trajectories of DLM spiking during different motor patterns. Insets: Representative traces illustrating different spiking activities associated with the distinct trajectories occupying different regions of the ISI ${ }^{-1}-\mathrm{CV}_{2} \mathrm{plot}^{\text {. }}$ (A) Trajectories of 'native' spike patterns during flight and grooming. (B) Trajectories of electroconvulsive stimulation- and PTX-evoked seizure spike patterns. Arrow heads indicated trajectory direction during seizure discharges.

plots succinctly display the differences in spike interval variability among the three types of spike patterns. Because the Poincaré trajectories from individual spike trains cannot be readily combined to produce an 'average' ensemble trajectory, we utilized the instantaneous coefficient of variation $\mathrm{CV}_{2}$ ([52], Figure 4B) to compile the merged, ensemble spike interval variability across a collection of spike trains that presented an overall signature of the temporal progression of spike interval variability for each motor program. It should be noted that $\mathrm{CV}_{2}$ documents a concise measure of variability of inter-spike intervals (e.g. [98, 99]), and can be derived from the Poincaré trajectory. Indeed, plots of the $\mathrm{ISI}^{-1} \mathrm{vs}$ $\mathrm{CV}_{2}$ trajectory of spike trains captured the salient features of the sustained rhythmic firing (low $\mathrm{CV}_{2}$ ) in flight spike trains, brief bouts of high-frequency firing during grooming (high $\mathrm{CV}_{2}$, variable ISI ${ }^{-1}$ ), and the characteristic progression of firing frequency and spike interval variability during delayed seizure discharges (Figure 10).

\section{Neurotransmitter Systems in Shaping Seizure Dis- charges and Native Motor Patterns}

Despite their obvious importance, a more comprehensive characterization remains to be accomplished to reveal the involvement of individual neurotransmitter systems in generating flight, grooming, and ECS-related activity in Drosophila. We aimed to provide an initial glimpse of the general vulnerability of the respective motor patterns to perturbations of excitatory, inhibitory and electrical transmission systems. The results also provide a context for the modulatory role of biogenic amines on these motor programs that have been examined to some depth [100, 80, 101]. We selected mutant alleles with well-described perturbations of neurotransmission. Specifically the Cha and ShakB flies displayed clear disruptions of cholinergic and electrical transmission respectively along GF pathway [41, 42], a robust neuronal circuit critical for escape behaviors [32, 33, 35, 36, 78]. For the $R d l$ mutant, the insecticide resistance phenotype has led to identification of the $\mathrm{GABA}_{\mathrm{A}}$ receptor gene and this particular allele that has been extensively studied [67]. Studies in cultured neurons have demonstrated altered sensitivity to $\mathrm{GABA}_{\mathrm{A}}$ blockade $[65,102]$.

Given the importance of these synaptic transmission systems on activity in the nervous system, it was somewhat unexpected that relatively minor modifications to flight and grooming patterning were uncovered (Figure 5B-C). Indeed, we observed that these mutant flies were capable of flight and grooming. Due to the intrinsically high variability across grooming spike trains, it is difficult to conclusively quantify any minor differences caused by the mutations. During flight, 
however, it was clear that only $R d l$ mutants displayed a reduction in DLM firing frequency, supporting the hypothesis of direct feedback regulation among DLM motor neurons underlying flight patterning [29, 53]. Conceivably, such feedback interactions may be mediated via GABAergic interneurons.

ECS discharges, in contrast to flight and grooming motor activities, appeared to be quite sensitive to disruption of neurotransmission systems. Interestingly, perturbations to GABAergic, cholinergic and electric synaptic systems each led to alterations in overlapping but not identical subsets of DD parameters (Figures 5-7). We found that enhancing GABAergic effectiveness in the $R d l$ allele and suppressing cholinergic transmission in Cha mutations severely reduced the peak firing frequency of delayed seizure discharges (Figure 5A), while $C h a$ and the gap junction mutant $S h a k B$ displayed increases in the threshold to induce a DD seizure with a corresponding shortened DD duration. Consistent with their integral role of transmission along the GF pathway, mutations in these transmission systems also prolonged the period of circuit failure following the high-frequency electroconvulsion stimulation. In particular, none of the ShakB mutants recovered over the $>80 \mathrm{~s}$ recording period (Figure 6). Notably, our analysis of DD in ShakB corroborates findings of a previous report of increased seizure thresholds in the same mutant alleles (Song $\&$ Tanouye, 2006). The alterations in spike patterning during DD were also evident in that $R d l$ and $S h a k B$ displayed more variable Poincaré plots and distinct ISI ${ }^{-1}$ vs $\mathrm{CV}_{2}$ trajectories compared to WT and Cha counterparts (Figure 7).

\section{Distinct Modes of Seizure Discharges Induced by Electroconvulsive Stimulation and GABAergic Block- ade}

Electroconvulsive stimulation and proconvulsant administration are two means for seizure induction commonly used in both vertebrates and invertebrates [103]. Several studies have utilized the proconvulsant picrotoxin (PTX), a noncompetitive $\mathrm{GABA}_{\mathrm{A}}$ antagonist, to induce seizure activity in Drosophila [21, 104, 105], However, the resulting seizure discharge pattern was reported to be qualitatively distinct from the ECS discharge repertoire [21]. We found that PTX application evoked a stereotypic, evolving DLM activity patterns (Figures 5-6). Unlike DD which occurred over a discrete period ( $\sim 15 \mathrm{~s})$, PTX-evoked activity continued throughout the recording period $(>1 \mathrm{hr}$ ), invoking the impression of the release or run-away of certain native, patterned activity. Initially, the DLM displayed a spontaneous, rhythmic spiking pattern reminiscent of flight activity in terms of firing frequency and the phase relation between left and right DLM spiking (compare Figure 4 'flight' and Figure 8 'flight-like'). This mode of activity was succeeded by bursts of spikes with instantaneous firing frequencies exceeding $100 \mathrm{~Hz}$ during which spiking was generally synchronized between the left and right sides. Loss of central inhibition is known to release a number of motor programs in insects [106], with a notable example of decapitation-triggered courtship motor programs in preying mantids [107]. Therefore, it is tempting to speculate that automatisms observed in the present study during ECSand PTX-induced seizures may represent two separate forms of synchronized activities 'released' by specific treatments $[108,109,110]$.

As summarized in Figure 10B, the $\mathrm{ISI}^{-1}$ vs. $\mathrm{CV}_{2}$ plot of the spike trains elucidates distinctions between DD and PTX-evoked activity indicating separate neurophysiological dysfunctions. The distinction between electrically and pharmacologically triggered discharges has also been noted in vertebrate seizure models. Indeed, the expression of seizure-like behavioral sequence evoked by pentylenetetrazole $\left(\mathrm{a} \mathrm{GABA}_{\mathrm{A}}\right.$ receptor antagonist) is distinct from seizure discharges evoked by maximal electroconvulsive stimulation in rats $[111,112]$.

These results serve to illustrate the importance to provide precise descriptions of seizure induction methods to avoid confusion in the literature when studying the molecular and cellular mechanisms in generating motor phenotypes. Furthermore, in future neurogenetic studies of 'seizure' behavior in Drosophila, a combination of pharmacological and electrical seizure induction methods can enhance the latitude and depth of analysis for the roles of individual genes participating in distinct mechanisms underpinning seizure activity.

Our study also demonstrates that in conjunction with spike firing rate and frequency distribution analyses, Poincaré plots adequately characterize the ISI variation along individual spike trains. The stochastic nature of spike trains, with ISI sequences varying from trial to trial within the same fly and between individuals, some inherent features characteristic of a particular motor pattern may be masked or overlooked. Nevertheless, the ability to construct $\mathrm{CV}_{2}$ for individual spike trains, which allows to derive an averaged ensemble trajectory pooled from a population flies, may serve as signatures in the $\mathrm{ISI}^{-1}$ vs. $\mathrm{CV}_{2}$ plot for the various motor patterns and to delineate how they are modified by genetic or pharmacological manipulations.

\section{Acknowledgments}

We thank members of the Wu Lab for their helpful comments over the course of this project. This work was supported by NIH grants: GM080255, GM088804, AG047612, and NS098590 to CFW, and NS082001 to AI

\section{References}

[1] W Kaplan and W Trout. The behavior of four neurological mutants of Drosophila. Genetics, 61(2):399, 1969.

[2] S Benzer. From the gene to behavior. J Am Med Assoc, 218(7):1015-1022, 1971.

[3] B Judd, M Shen, and T Kaufman. The anatomy and function of a segment of the $\mathrm{x}$ chromosome of drosophila melanogaster. Genetics, 71(1):139-156, 1972. 
[4] T Grigliatti, L Hall, R Rosenbluth, and D Suzuki. Temperature-sensitive mutations in drosophila melanogaster. Mol Gen Genet, 120(2):107-114, 1973.

[5] F Jackson, S Wilson, G Strichartz, and L Hall. Two types of mutants affecting voltage-sensitive sodium channels in drosophila melanogaster. Nature, 308(5955):189, 1984.

[6] B Ganetzky and C-F Wu. Indirect suppression involving behavioral mutants with altered nerve excitability in drosophila melanogaster. Genetics, 100(4):597-614, 1982.

[7] R Williamson. Lithium stops hereditary shuddering in drosophila melanogaster. Psychopharmacology, 76(3):265-268, 1982.

[8] K Loughney, R Kreber, and B Ganetzky. Molecular analysis of the para locus, a sodium channel gene in drosophila. Cell, 58(6):1143-1154, 1989.

[9] S Titus, J Warmke, and B Ganetzky. The drosophila erg $\mathrm{k}+$ channel polypeptide is encoded by the seizure locus. J Neurosci, 17(3):875-881, 1997.

[10] F Kawasaki, R Felling, and R Ordway. A temperaturesensitive paralytic mutant defines a primary synaptic calcium channel in drosophila. J Neurosci, 20(13):48854889, 2000.

[11] R Marley and R Baines. Increased persistent na+ current contributes to seizure in the slamdance bang-sensitive drosophila mutant. J Neurophysiol, 106(1):18, 2011.

[12] M Palladino, J Bower, R Kreber, and B Ganetzky. Neural dysfunction and neurodegeneration indrosophila nat/k+ atpase alpha subunit mutants. J Neurosci, 23(4):12761286, 2003.

[13] D Hekmat-Scafe, M Lundy, R Ranga, and M Tanouye. Mutations in the $\mathrm{k}+/ \mathrm{cl}$ - cotransporter gene kazachoc (kcc) increase seizure susceptibility in drosophila. $J$ Neurosci, 26(35):8943-8954, 2006.

[14] J Melom and J Littleton. Mutation of a nckx eliminates glial microdomain calcium oscillations and enhances seizure susceptibility. J Neurosci, 33(3):1169-1178, 2013.

[15] C Royden, V Pirrotta, and L Jan. The tko locus, site of a behavioral mutation in d. melanogaster, codes for a protein homologous to prokaryotic ribosomal protein s12. Cell, 51(2):165-173, 1987.

[16] H Zhang, J Tan, E Reynolds, D Kuebler, S Faulhaber, and $\mathrm{M}$ Tanouye. The drosophila slamdance gene: a mutation in an aminopeptidase can cause seizure, paralysis and neuronal failure. Genetics, 162(3):1283-1299, 2002.

[17] T Fergestad, B Bostwick, and B Ganetzky. Metabolic disruption in drosophila bang-sensitive seizure mutants. Genetics, 173(3):1357-1364, 2006.
[18] N Trotta, C Rodesch, T Fergestad, and K Broadie. Cellular bases of activity-dependent paralysis in drosophila stress-sensitive mutants. J Neurobiol, 60(3):328-347, 2004.

[19] L Salkoff and L Kelly. Temperature-induced seizure and frequency-dependent neuromuscular block in a ts mutant of drosophila. Nature, 273(5658):156, 1978.

[20] $P$ Pavlidis and $M$ Tanouye. Seizures and failures in the giant fiber pathway of drosophila bang-sensitive paralytic mutants. J Neurosci, 15(8):5810-5819, 1995.

[21] J Lee and C-F Wu. Electroconvulsive seizure behavior in drosophila: analysis of the physiological repertoire underlying a stereotyped action pattern in bang-sensitive mutants. J Neurosci, 22(24):11065-11079, 2002.

[22] J Lee and C-F Wu. Genetic modifications of seizure susceptibility and expression by altered excitability in drosophila na+ and k+ channel mutants. J Neurophysiol, 96(5):2465-2478, 2006.

[23] D Kuebler, H Zhang, X Ren, and M Tanouye. Genetic suppression of seizure susceptibility in drosophila. $J$ Neurophysiol, 86(3):1211-1225, 2001.

[24] A Ghezzi, J Pohl, Y Wang, and N Atkinson. Bk channels play a counter-adaptive role in drug tolerance and dependence. Proc Natl Acad Sci USA, 107(37):16360-16365, 2010.

[25] S Ehaideb, A Iyengar, A Ueda, G Iacobucci, C Cranston, A Bassuk, D Gubb, J Axelrod, S Gunawardena, C-F Wu, et al. prickle modulates microtubule polarity and axonal transport to ameliorate seizures in flies. Proc Natl Acad Sci USA, 111(30):11187-11192, 2014.

[26] M Dickinson and M Tu. The function of dipteran flight muscle. Comp Biochem and Physiol A, 116(3):223-238, 1997.

[27] A Iyengar and C-F Wu. Flight and seizure motor patterns in drosophila mutants: simultaneous acoustic and electrophysiological recordings of wing beats and flight muscle activity. J Neurogenet, 28(3-4):316-328, 2014.

[28] J Levine and R Wyman. Neurophysiology of flight in wild-type and a mutant drosophila. Proc Natl Acad Sci USA, 70(4):1050-1054, 1973.

[29] E Harcombe and R Wyman. Output pattern generation by drosophila flight motoneurons. J Neurophysiol, 40(5):1066-1077, 1977.

[30] S Gordon and M Dickinson. Role of calcium in the regulation of mechanical power in insect flight. Proc Natl Acad Sci USA, 103(11):4311-4315, 2006.

[31] F-O Lehmann, D Skandalis, and R Berthé. Calcium signalling indicates bilateral power balancing in the drosophila flight muscle during manoeuvring flight. Journal of The Royal Society Interface, 10(82):20121050, 2013. 
[32] M Tanouye and R Wyman. Motor outputs of giant nerve fiber in drosophila. J Neurophsyiol, 44(2):405-421, 1980.

[33] J Trimarchi and A Schneiderman. Different neural pathways coordinate drosophila flight initiations evoked by visual and olfactory stimuli. J Exp Biol, 198(5):10991104, 1995.

[34] J Engel and C-F Wu. Interactions of membrane excitability mutations affecting potassium and sodium currents in the flight and giant fiber escape systems of drosophila. J Comp Physiol A, 171(1):93-104, 1992.

[35] J Engel and C-F Wu. Altered habituation of an identified escape circuit in drosophila memory mutants. J Neurosci, 16(10):3486-3499, 1996.

[36] G Card and M Dickinson. Visually mediated motor planning in the escape response of drosophila. Curr Biol, 18(17):1300-1307, 2008.

[37] A Ewing. The neuromuscular basis of courtship song indrosophila: The role of the indirect flight muscles. $J$ Comp Physiol, 119(3):249-265, 1977.

[38] A Takeuchi and N Takeuchi. A study of the action of picrotoxin on the inhibitory neuromuscular junction of the crayfish. J Physiol, 205(2):377-391, 1969.

[39] R Ffrench-Constant, D Mortlock, C Shaffer, R MacIntyre, and R Roush. Molecular cloning and transformation of cyclodiene resistance in drosophila: an invertebrate gamma-aminobutyric acid subtype a receptor locus. Proc Natl Acad Sci USA, 88(16):7209-7213, 1991.

[40] R Greenspan, J Finn Jr, and J Hall. Acetylcholinesterase mutants in drosophila and their effects on the structure and function of the cental nervous system. J Comp Neurol, 189(4):741-774, 1980.

[41] M Gorczyca and J Hall. Identification of a cholinergic synapse in the giant fiber pathway of drosophila using conditional mutations of acetylcholine synthesis. $\mathrm{J} \mathrm{Neu}$ rogenet, 1(4):289-313, 1984.

[42] J Thomas and R Wyman. Mutations altering synaptic connectivity between identified neurons in drosophila. $J$ Neurosci, 4(2):530-538, 1984.

[43] A Frankel and G Brousseau. Drosophila medium that does not require dried yeast. Drosophila Inf Serv, 43:184, 1968.

[44] R Greenspan. Mutations of choline acetyltransferase and associated neural defects. J Comp Physiol, 137(1):8392, 1980.

[45] T Homyk Jr and T Grigliatti. Behavioral mutants of drosophila melanogaster. iv. analysis of developmentally temperature-sensitive mutations affecting flight. Dev Genet, 4(2):77-97, 1983.

[46] Dan L Lindsley and Georgianna G Zimm. The genome of Drosophila melanogaster. Academic Press, 1992.
[47] A Miller. The internal anatomy and histology of the imago Drosophila melanogaster.

[48] I Howlett and M Tanouye. Seizure-sensitivity in drosophila is ameliorated by dorsal vessel injection of the antiepileptic drug valproate. J Neurogenet, 27(4):143-150, 2013.

[49] B Knight. Dynamics of encoding in a population of neurons. J Gen Phsyiol, 59(6):734-766, 1972.

[50] P Lánskỳ, R Rodriguez, and L Sacerdote. Mean instantaneous firing frequency is always higher than the firing rate. Neural computation, 16(3):477-489, 2004.

[51] G Werner and V Mountcastle. The variability of central neural activity in a sensory system, and its implications for the central reflection of sensory events. J Neurophysiol, 26(6):958-977, 1963.

[52] G Holt, W Softky, C Koch, and R Douglas. Comparison of discharge variability in vitro and in vivo in cat visual cortex neurons. J Neurophysiol, 75(5):1806-1814, 1996.

[53] J Koenig and K Ikeda. Interspike interval relationship among flight muscle fibres in drosophila. J Exp Biol, 87(1):137-147, 1980.

[54] J Curtsinger and C Laurie-Ahlberg. Genetic variability of flight metabolism in drosophila melanogaster. i. characterization of power output during tethered flight. Genetics, 98(3):549-564, 1981.

[55] F-O Lehmann and M Dickinson. The changes in power requirements and muscle efficiency during elevated force production in the fruit fly drosophila melanogaster. $J$ Exp Biol, 200(7):1133-1143, 1997.

[56] R Phillis, A Bramlage, C Wotus, A Whittaker, L Gramates, D Seppala, F Farahanchi, P Caruccio, and R Murphey. Isolation of mutations affecting neural circuitry required for grooming behavior in drosophila melanogaster. Genetics, 133(3):581-592, 1993.

[57] A Seeds, P Ravbar, P Chung, S Hampel, F Midgley Jr, B Mensh, and J Simpson. A suppression hierarchy among competing motor programs drives sequential grooming in drosophila. Elife, 3:e02951, 2014.

[58] J Coggshall. Neurons associated with the dorsal longitudinal flight muscles ofdrosophila melanogaster. J Comp Neurol, 177(4):707-720, 1978.

[59] A Longtin. Stochastic resonance in neuron models. $J$ Stat Phys, 70(1-2):309-327, 1993.

[60] A Szúcs. Applications of the spike density function in analysis of neuronal firing patterns. J Neurosci Method, 81(1-2):159-167, 1998.

[61] M Brennan, M Palaniswami, and P Kamen. Do existing measures of poincare plot geometry reflect nonlinear features of heart rate variability? IEEE trans biomed eng, 48(11):1342-1347, 2001. 
[62] T Homyk, J Szidonya, and D Suzuki. Behavioral mutants of drosophila melanogaster. Mol Gen Genet, 177(4):553-565, 1980.

[63] D Crompton, A Griffin, J Davies, and G Miklos. Analysis of a cdna from the neurologically active locus shaking-b (passover) of drosophila melanogaster. Gene, 122(2):385-386, 1992.

[64] P Phelan, L Stebbings, R Baines, J Bacon, J Davies, and C Ford. Drosophila shaking-b protein forms gap junctions in paired xenopus oocytes. Nature, 391(6663):181, 1998.

[65] H-G Zhang, M Jackson, et al. A unique amino acid of the drosophila gaba receptor with influence on drug sensitivity by two mechanisms. J Physiol, 479(1):65-75, 1994.

[66] C-T Wang, H-G Zhang, T Rocheleau, M Jackson, et al. Cation permeability and cation-anion interactions in a mutant gaba-gated chloride channel from drosophila. Biophys J, 77(2):691-700, 1999.

[67] R ffrench Constant, M Williamson, $T$ Davies, and $\mathrm{C}$ Bass. Ion channels as insecticide targets. $\mathrm{J} \mathrm{Neu}$ rogenet, 30(3-4):163-177, 2016.

[68] P Phelan, M Nakagawa, M Wilkin, K Moffat, C O'Kane, J Davies, and J Bacon. Mutations in shaking-b prevent electrical synapse formation in the drosophila giant fiber system. J Neurosci, 16(3):1101-1113, 1996.

[69] Ligong Chen, Kathleen A Durkin, and John E Casida. Structural model for $\gamma$-aminobutyric acid receptor noncompetitive antagonist binding: widely diverse structures fit the same site. Proceedings of the National Academy of Sciences, 103(13):5185-5190, 2006.

[70] T Miller. Structure and physiology of the circulatory system. Comp Insect physiol, biochem and pharmacol, 3:289-353, 1985.

[71] O Siddiqi and S Benzer. Neurophysiological defects in temperature-sensitive paralytic mutants of drosophila melanogaster. Proc Natl Acad Sci USA, 73(9):32533257, 1976.

[72] L Salkoff and R Wyman. Genetic modification of potassium channels in drosophila shaker mutants. Nature, 293(5829):228, 1981.

[73] T Elkins, B Ganetzky, and C-F Wu. A drosophila mutation that eliminates a calcium-dependent potassium current. Proc Natl Acad Sci USA, 83(21):8415-8419, 1986.

[74] J Engel and C-F Wu. Genetic dissection of functional contributions of specific potassium channel subunits in habituation of an escape circuit indrosophila. J Neurosci, 18(6):2254-2267, 1998.

[75] J Engel, X-J Xie, M Sokolowski, and C-F Wu. A cgmp-dependent protein kinase gene, foraging, modifies habituation-like response decrement of the giant fiber escape circuit in drosophila. Learning \& Memory, 7(5):341-352, 2000.

[76] D Kadas, A Tzortzopoulos, E Skoulakis, and C Consoulas. Constitutive activation of ca2+/calmodulindependent protein kinase ii during development impairs central cholinergic transmission in a circuit underlying escape behavior in drosophila. J Neurosci, 32(1):170$182,2012$.

[77] L Mu, K Ito, J Bacon, and N Strausfeld. Optic glomeruli and their inputs in drosophila share an organizational ground pattern with the antennal lobes. J Neurosci, 32(18):6061-6071, 2012.

[78] C Von Reyn, P Breads, M Peek, G Zheng, W Williamson, A Yee, A Leonardo, and G Card. A spike-timing mechanism for action selection. Nat Neurosci, 17(7):962, 2014.

[79] T Elkins and B Ganetzky. The roles of potassium currents in drosophila flight muscles. J Neurosci, 8(2):428434, 1988.

[80] B Brembs, F Christiansen, H Pflüger, and C Duch. Flight initiation and maintenance deficits in flies with genetically altered biogenic amine levels. J Neurosci, 27(41):11122-11131, 2007.

[81] J Melzig, S Buchner, F Wiebel, R Wolf, E Buchner, M Burg, and WL Pak. Genetic depletion of histamine from the nervous system of drosophila eliminates specific visual and mechanosensory behavior. J Comp Physiol A, 179(6):763-773, 1996.

[82] D Kuebler and M Tanouye. Modifications of seizure susceptibility in drosophila. J Neurophysiol, 83(2):9981009, 2000.

[83] L Parker, M Padilla, Y Du, K Dong, and M Tanouye. Drosophila as a model for epilepsy: bss is a gain-offunction mutation in the para sodium channel gene that leads to seizures. Genetics, 187(2):523-534, 2011.

[84] D King and R Wyman. Anatomy of the giant fibre pathway indrosophila. i. three thoracic components of the pathway. J Neurocytol, 9(6):753-770, 1980.

[85] K Ikeda and J Koenig. Morphological identification of the motor neurons innervating the dorsal longitudinal flight muscle of drosophila melanogaster. J Comp Neurol, 273(3):436-444, 1988.

[86] J Fernandes, M Bate, and K Vijayraghavan. Development of the indirect flight muscles of drosophila. Development, 113(1):67-77, 1991.

[87] C Consoulas, L Restifo, and R Levine. Dendritic remodeling and growth of motoneurons during metamorphosis of drosophila melanogaster. J Neurosci, 22(12):49064917, 2002.

[88] S Hebbar and J Fernandes. Pruning of motor neuron branches establishes the dlm innervation pattern in drosophila. J Neurobiol, 60(4):499-516, 2004. 
[89] S Ryglewski, L Kilo, and C Duch. Sequential acquisition of cacophony calcium currents, sodium channels and voltage-dependent potassium currents affects spike shape and dendrite growth during postembryonic maturation of an identified d rosophila motoneuron. Eur $J$ Neurosci, 39(10):1572-1585, 2014.

[90] S Ryglewski, K Lance, R Levine, and C Duch. Cav2 channels mediate low and high voltage-activated calcium currents in drosophila motoneurons. J Physiol, 590(4):809-825, 2012.

[91] S Ryglewski and C Duch. Shaker and shal mediate transient calcium-independent potassium current in a drosophila flight motoneuron. J Neurophysiol, 102(6):3673-3688, 2009.

[92] M Herrera-Valdez, E McKiernan, S Berger, S Ryglewski, $\mathrm{C}$ Duch, and S Crook. Relating ion channel expression, bifurcation structure, and diverse firing patterns in a model of an identified motor neuron. J Comput Neurosci, 34(2):211-229, 2013.

[93] R Wyman. Probabilistic characterization of simultaneous nerve impulse sequences controlling dipteran flight. Biophys J, 5(4):447-471, 1965.

[94] W Liu and R Wilson. Glutamate is an inhibitory neurotransmitter in the drosophila olfactory system. Proc Natl Acad Sci USA, 110(25):10294-10299, 2013.

[95] A Goldberger, D Rigney, and B West. Chaos and fractals in human physiology. Sci Am, 262(2):42-49, 1990.

[96] H Huikuri, T Makikallio, C-K Peng, A Goldberger, U Hintze, and M Møller. Fractal correlation properties of rr interval dynamics and mortality in patients with depressed left ventricular function after an acute myocardial infarction. Circulation, 101(1):47-53, 2000.

[97] O Akerberg and M Chacron. In vivo conditions influence the coding of stimulus features by bursts of action potentials. J Comput Neurosci, (2):369-383, 2011.

[98] A Bacci and J Huguenard. Enhancement of spiketiming precision by autaptic transmission in neocortical inhibitory interneurons. Neuron, 49(1):119-130, 2006.

[99] X Chen, P Green, and J Levine. Stress enhances muscle nociceptor activity in the rat. Neuroscience, 185:166173, 2011.

[100] C Yellman, H Tao, B He, and J Hirsh. Conserved and sexually dimorphic behavioral responses to biogenic amines in decapitated drosophila. Proc Natl Acad Sci USA, 94(8):4131-4136, 1997.

[101] Sufia Sadaf, O Venkateswara Reddy, Sanjay P Sane, and Gaiti Hasan. Neural control of wing coordination in flies. Curr Biol, 25(1):80-86, 2015.

[102] D Lee, H Su, and D O'Dowd. Gaba receptors containing rdl subunits mediate fast inhibitory synaptic transmission in drosophila neurons. J Neurosci, 23(11):4625-4634, 2003.
[103] W Löscher and D Schmidt. Which animal models should be used in the search for new antiepileptic drugs? a proposal based on experimental and clinical considerations. Epilepsy Res, 2(3):145-181, 1988.

[104] Geoff E Stilwell, Sudipta Saraswati, J Troy Littleton, and Scott W Chouinard. Development of a drosophila seizure model for in vivo high-throughput drug screening. Eur J Neurosci, 24(8):2211-2222, 2006.

[105] C Giachello and R Baines. Inappropriate neural activity during a sensitive period in embryogenesis results in persistent seizure-like behavior. Curr Biol, 25(22):29642968, 2015.

[106] B Harris, Rand Pfeiffer, G Rubin, and J Truman. Neuron hemilineages provide the functional ground plan for the drosophila ventral nervous system. Elife, 4:e04493, 2015.

[107] K Roeder. An experimental analysis of the sexual behavior of the praying mantis (mantis religiosa 1.). Biol Bull, 69(2):203-220, 1935.

[108] H Jasper. Some physiological mechanisms involved in epileptic automatisms. Epilepsia, 5(1):1-20, 1964.

[109] S Geier, J Bancaud, J Talairach, A Bonis, M Enjelvin, and $\mathrm{H}$ Hossard-Bouchaud. Automatisms during frontal lobe epileptic seizures. Brain, 99(3):447-458, 1976.

[110] S Noachtar and A Peters. Semiology of epileptic seizures: a critical review. Epilepsy \& Behav, 15(1):2-9, 2009.

[111] R Racine. Modification of seizure activity by electrical stimulation: Ii. motor seizure. Electroencephalograph Clin Neurophysiol, 32(3):281-294, 1972.

[112] A Lüttjohann, P Fabene, and G van Luijtelaar. A revised racine's scale for ptz-induced seizures in rats. Physiol \& Behav, 98(5):579-586, 2009. 